\title{
Classes of sum-of-cisoids processes and their statistics for the modeling and simulation of mobile fading channels
}

\author{
Bjørn Olav Hogstad ${ }^{1 *}$, Carlos A Gutiérrez ${ }^{2}$, Matthias Pätzold ${ }^{3}$ and Pedro M Crespo ${ }^{1}$
}

\begin{abstract}
In this paper, we present a fundamental study on the stationarity and ergodicity of eight classes of sum-of-cisoids (SOC) processes for the modeling and simulation of frequency-nonselective mobile Rayleigh fading channels. The purpose of this study is to determine which classes of SOC models enable the design of channel simulators that accurately reproduce the channel's statistical properties without demanding information on the time origin or the time-consuming computation of an ensemble average. We investigate the wide-sense stationarity, first-order stationarity of the envelope, mean ergodicity, and autocorrelation ergodicity of the underlying random processes characterizing the different classes of stochastic SOC simulators. The obtained results demonstrate that only the class of SOC models comprising cisoids with constant gains, constant frequencies, and random phases is defined by a set of stationary and ergodic random processes. The analysis presented here can easily be extended with respect to the modeling and simulation of frequency-selective single-input single-output (SISO) and multiple-input multiple-output channels. For the case of frequency-selective SISO channels, we investigate the stationarity and ergodicity in both time and frequency of 16 different classes of SOC simulation models. The findings presented in this paper can be used in the laboratory as guidelines to design efficient simulation platforms for the performance evaluation of modern mobile communication systems.
\end{abstract}

\section{Introduction}

Computer simulators have become fundamental tools for the design, test, and optimization of modern mobile communication systems [1]. They provide a powerful, affordable, and reproducible means to assess the system performance. An important component of any computer simulator employed for system analysis is the model chosen to simulate the channel. This is indeed a critical component as most of the problems affecting the performance of wireless communication systems, e.g., intersymbol interference and cross modulation, are caused by the channel [2]. There exist several different approaches to the design of fading channel simulators, such as those described in [3-6]. While the underlying methodology may differ, the principal objective of all stochastic channel simulators is the same: synthesizing a random process

\footnotetext{
*Correspondence: bohogstad@ceit.es

${ }^{1}$ CEIT and Tecnun, University of Navarra, Manuel de Lardizábal 15, San

Sebastián 20018, Spain

Full list of author information is available at the end of the article
}

that can efficiently be realized on a software or hardware simulation platform under the constraint that its statistical properties resemble those of a nonrealizable reference channel model. A typical nonrealizable reference channel model is, for example, the Rayleigh fading channel model under isotropic scattering conditions ([7], Sec. 2.1.2). A realizable random process constitutes the so-called channel simulation model ([8], Sec. 4.1).

Two often desirable properties of any stochastic simulation model for small-scale multipath radio channels are stationarity and ergodicity. These properties enable the channel simulator to accurately emulate the channel's statistical properties on the basis of a single simulation run (ergodicity) without requiring information on the time origin (stationarity). In the strict sense, a channel simulator is stationary if all marginal and joint probability density functions (PDFs) of the underlying random process characterizing the simulation model are independent of a time-shift ([9], pp. 297-303). On the other hand,

\section{Springer}

(c) 2013 Hogstad et al: licensee Springer. This is an Open Access article distributed under the terms of the Creative Commons Attribution License (http://creativecommons.org/licenses/by/2.0), which permits unrestricted use, distribution, and reproduction in any medium, provided the original work is properly cited. 
a channel simulator is ergodic if the time averages of the simulation model are equal to the ensemble averages ([9], Sec. 13.1). These conditions are too stringent and can hardly be met in practice. However, the information about the channel's statistics of order higher than 2 is rarely required to assess the performance of wireless communication systems. Hence, for most practical purposes, it suffices if the channel simulator is wide-sense stationary (WSS) and ergodic with respect to (w.r.t.) the mean value and the autocorrelation function (ACF). In [10,11], the advantages of ergodic channel simulators versus nonergodic simulation models for the performance analysis w.r.t. the bit error probability have been demonstrated. More specifically, in [10], this observation has been done for noncoherent differential phase shift keying since the bit error probability depends on the ACF of the underlying complex Gaussian process. If the channel simulator is nonergodic, time ACF of the simulation model does not fit well with ensemble ACF of the reference model. In this case, one needs to average over different realizations of the underlying stochastic process to increase the performance, which also drastically increases the complexity of the channel simulator. Indeed, an important aspect of the statistical characterization of a channel simulator consists in determining whether the simulation model is a WSS, a mean-ergodic (ME), or an autocorrelation-ergodic (AE) random process. For some applications, such as the evaluation of diversity systems ([7], Ch. 6), it is also very important to know whether the envelope of the simulation model is a first-order stationary (FOS) process. An interesting study of the relation between the coherence interval and the spectral spread of a zero-mean WSS process and its application to mobile fading channels can be found in [12]. In addition, an investigation of the time interval within which the fading process is expected to fulfill the stationarity assumption in real-world channels can be found in $[13,14]$.

Among the variety of channel simulation models proposed in the literature, those based on Rice's sum-ofsinusoids (SOS) principle $[15,16]$ have widely been in use as a basis for the design of multipath radio channel simulators (e.g., see [17-21]). In the conventional SOS channel simulation approach, it is assumed that the inphase and quadrature (IQ) components of the channel's complex envelope are statistically independent Gaussian processes. Under this consideration, the simulation of the channel's IQ components is carried out by means of two uncorrelated SOS processes having disjoint sets of parameters - gains, frequencies, phases, and number of sinusoids. This approach has been applied for over four decades to the simulation of a variety of mobile fading channels, ranging from simple single-input single-output (SISO) channels [18] to sophisticated multiple-input multipleoutput (MIMO) channels [19]. It is important to notice that the conventional SOS simulation approach suffers from a serious limitation, that is, it can only be used to simulate fading channels characterized by symmetrical Doppler power spectral densities (DPSDs), leaving aside the more realistic case of channels having asymmetrical DPSDs. ${ }^{\text {a }}$ A solution to overcome this limitation provides a variation of the conventional SOS approach, where a finite sum of complex sinusoids (cisoids) is used to simulate the channel's complex envelope [22]. Basically, SOC models differ from conventional SOS models such that the IQ components of the former models are characterized by the same set of parameters [22]. This feature of SOC models enables the simulation of fading channels having symmetrical and asymmetrical DPSDs [23-26]. Another important feature of SOC models is that they have a clear physical meaning as they are related to the electromagnetic plane wave model $[8,27]$. This is in contrast to the conventional SOS model, which cannot be interpreted physically.

Despite the significant attention that SOC channel simulation models have attracted, a comprehensive study on their stationary and ergodic properties is still lacking in the literature. To close this gap, we present in this paper a systematic analysis of the wide-sense stationarity, firstorder stationarity of the envelope, mean ergodicity, and autocorrelation ergodicity of eight fundamental classes of SOC simulation models for frequency-nonselective SISO Rayleigh fading channels. This analysis can easily be extended with respect to the modeling and simulation of frequency-selective SISO and MIMO channels. For the case of frequency-selective SISO channels, we provide in this paper a concise investigation of the stationarity and ergodicity in both time and frequency of 16 different classes of SOC simulation models. The presented results are intended to serve as guidelines for the design of efficient channel simulators for the performance evaluation of mobile communication systems.

The rest of the paper is organized as follows. In Section 2, we discuss the related work and highlight the novelty and importance of our paper. In Sections 3 and 4, we briefly review the reference model and the SOC model, respectively. In Section 5, we summarize the characteristics of stationary and ergodic processes. Section 6 introduces eight classes of SOC-based simulation models and investigates their stationary and ergodic properties. An extension to frequency-selective channels can be found in Section 7. A discussion of the obtained results and their applications to widely used parameter computation methods can be found in Section 8. Finally, the conclusions are drawn in Section 9.

\section{Related work}

Even though the stationary and ergodic properties of SOC channel simulation models are not fully known, those 
of conventional SOS simulators have thoroughly been analyzed in [28-30]. In these papers, the authors have analyzed the ME and AE properties of eight classes of deterministic and stochastic SOS simulation models for Rayleigh fading channels. They also investigated the widesense stationarity and the first-order stationarity of the IQ components of all eight classes of SOS channel simulators. It is shown in [28-30] that an SOS process is stationary and ergodic if and only if the sinusoids' gains and Doppler frequencies are constant quantities and the phases are random variables. We notice, nonetheless, that the conclusions drawn in these papers cannot be extrapolated to the case of SOC simulators. The reason is that the investigations performed in [28-30] were carried out in the framework of the conventional SOS channel simulation approach, implying the condition that the IQ components of the channel simulation model are uncorrelated. This condition does not necessarily hold for SOC simulators, meaning that their IQ components are in general correlated [31]. It is precisely this particular feature that enables SOC models to simulate fading channels characterized not only by symmetrical DPSDs but also by asymmetrical ones. It is therefore necessary to revisit the analysis in [28-30] to shed light on the peculiar and important case of SOC channel simulators. The results presented in this paper will demonstrate that the distribution of the envelope and the autocorrelation properties of SOC models differ completely from what is known from conventional SOS models.

\section{The reference model}

A Rayleigh process $\zeta(t)$ is defined as

$$
\zeta(t)=\left|\mu_{1}(t)+j \mu_{2}(t)\right|
$$

where $\mu_{1}(t)$ and $\mu_{2}(t)$ are zero-mean real Gaussian processes, each with variance $\sigma_{0}^{2}$. ${ }^{\mathrm{b}}$ The process $\mu(t)=$ $\mu_{1}(t)+j \mu_{2}(t)$ will be considered as a complex Gaussian process. In general, it is usually assumed that $\mu_{1}(t)$ and $\boldsymbol{\mu}_{2}(t)$ are uncorrelated, but in this paper, we have allowed them to be correlated. The correlation properties are described by the correlation matrix in ([32], eq. (17)). Here, a process $X(t)$ is called a complex Gaussian process if each finite-dimensional vector $\left[\boldsymbol{X}\left(t_{1}\right), \ldots, \boldsymbol{X}\left(t_{n}\right)\right]^{T}$ is a complex Gaussian vector. A complex random vector is said to be Gaussian if its real and imaginary parts are jointly Gaussian [33]. Therein, it is shown that if the process $\boldsymbol{\mu}(t)$ is proper (or circularly symmetric), the density of the mentioned complex Gaussian vector is completely described by the ACF $r_{\mu \mu}(\tau) \triangleq$ $E\left\{\boldsymbol{\mu}^{*}(t) \boldsymbol{\mu}(t+\tau)\right\}$.
According to the correlation model proposed by Clarke in [34] for two-dimensional scattering environments, the ACF $r_{\mu \mu}(\tau)$ of $\boldsymbol{\mu}(t)$ can be expressed as

$$
r_{\mu \mu}(\tau)=2 \sigma_{0}^{2} \int_{0}^{2 \pi} p_{\alpha}(\alpha) e^{j 2 \pi f_{\max } \cos (\alpha) \tau} d \alpha .
$$

In the equation above, $f_{\max }$ is referred to as the maximum Doppler frequency, and $p_{\alpha}(\alpha)$ denotes the density function of the angle of arrival (AOA) of the incoming waves.

\section{The SOC model}

It is well known (see, e.g., [35-37]) that Rayleigh fading channel models, characterized by the complex Gaussian process $\boldsymbol{\mu}(t)$, can be modeled and efficiently simulated using the SOC process

$$
\hat{\boldsymbol{\mu}}(t)=\sum_{n=1}^{N} c_{n} e^{j\left(2 \pi f_{n} t+\boldsymbol{\theta}_{n}\right)},
$$

where $N$ denotes the number of cisoids, $c_{n}$ is called the gain, $f_{n}$ designates the Doppler frequency, and $\boldsymbol{\theta}_{n}$ denominates the phase of the $n$th cisoid. The phases $\boldsymbol{\theta}_{n}$ are independent and identically distributed (i.i.d.) random variables, each following a uniform distribution over $[0,2 \pi)$. It is worth mentioning that the frequencies $f_{n}$ are related to the maximum Doppler frequency $f_{\max }$ and AOAs $\alpha_{n}$ of the waves that reach the receiver antenna by means of the transformation

$$
f_{n}=f_{\max } \cos \left(\alpha_{n}\right), \quad n=1, \ldots, N .
$$

These parameters have a strong influence on the temporal correlation properties of SISO channels and also on the spatial and temporal correlation properties of MIMO channels. Furthermore, in [25], it is shown that the cisoids' frequencies are directly related to the angles of departure of transmitted waves in mobile-to-mobile double Rayleigh fading channels.

Owing to the central limit theorem ([9], p. 278), the stochastic process $\hat{\boldsymbol{\mu}}(t)$ tends to the complex Gaussian process $\boldsymbol{\mu}(t)$ as $N \rightarrow \infty$. From the results obtained in [22], it is straightforward to show that the obtained process $\boldsymbol{\mu}(t)$ is proper. If the phases $\theta_{n}$ are outcomes (realizations) of a random generator with a uniform distribution in the interval $(0,2 \pi]$, then the stochastic process $\hat{\mu}(t)$ results in the sample function

$$
\tilde{\mu}(t)=\sum_{n=1}^{N} c_{n} e^{j\left(2 \pi f_{n} t+\theta_{n}\right)},
$$

which is completely deterministic.

The SOC model depends on three types of parameters, namely the gains $c_{n}$, the frequencies $f_{n}$, and the phases $\theta_{n}$. 
In principle, each type of these parameters can be introduced as a random variable or constant. Hence, altogether, $2^{3}=8$ classes of SOC models for Rayleigh fading channels can be defined. If all parameters are constants, we obtain a completely deterministic process, denoted by $\tilde{\zeta}(t)=$ $|\tilde{\mu}(t)|=\left|\tilde{\mu}_{1}(t)+j \tilde{\mu}_{2}(t)\right|$. As opposed to this, at least one random variable is required to obtain a stochastic process, denoted by $\hat{\boldsymbol{\zeta}}(t)=|\hat{\boldsymbol{\mu}}(t)|=\left|\hat{\boldsymbol{\mu}}_{1}(t)+j \hat{\boldsymbol{\mu}}_{2}(t)\right|$. For the performance evaluation of SOC simulation models with random/constant parameters, it is important to know the conditions for which the resulting stochastic process $\hat{\zeta}(t)$ is stationary. Also, it is important to know under which conditions the stochastic process $\hat{\mu}(t)$ results in an ergodic process. In the next section, we will review briefly the characteristics of stationary and ergodic processes.

\section{Stationarity and ergodicity}

A stochastic process $\hat{\zeta}(t)$ is called strict-sense stationary (SSS) ([9], p. 387) if its statistical properties are invariant to a shift of the origin. This means that the stochastic processes $\hat{\zeta}(t)$ and $\hat{\zeta}(t+c)$ have the same statistics for any $c \in \mathbb{R}$. It follows that the $m$ th-order density function must satisfy the equation

$$
\begin{aligned}
p_{\hat{\zeta}}\left(x_{1}, \ldots, x_{m} ; t_{1}, \ldots, t_{m}\right)=p_{\hat{\zeta}} & \left(x_{1}, \ldots, x_{m} ;\right. \\
& \left.t_{1}+c, \ldots, t_{m}+c\right),
\end{aligned}
$$

for all values of $t_{1}, \ldots, t_{m}$, and $c$.

A stochastic process $\hat{\zeta}(t)$ is called FOS ([9], p. 392) if the density of $\hat{\zeta}(t)$ satisfies

$$
p_{\hat{\zeta}}(x ; t)=p_{\hat{\zeta}}(x ; t+c),
$$

for all values of $t$ and $c$. This implies that the mean and the variance of $\hat{\zeta}(t)$ are independent of time. If a stochastic process is SSS, then the process is also FOS. The inverse statement is not always true. For simplicity and brevity, we only investigate the FOS properties of $\hat{\zeta}(t)$. A stochastic process $\hat{\zeta}(t)$ is called asymptotically FOS if the density $p_{\hat{\zeta}}(x ; t+c)$ tends to a limit, which is independent of $c$ as $c \rightarrow \infty$ ([9], p. 392).

A stochastic process $\hat{\mu}(t)$ is said to be WSS ([9], p. 388) if $\hat{\mu}(t)$ satisfies the following conditions:

1. The mean of $\hat{\boldsymbol{\mu}}(t)$ is constant, i.e.,

$$
E\{\hat{\boldsymbol{\mu}}(t)\}=m_{\hat{\mu}}=\text { const. }
$$

2. The ACF of $\hat{\mu}(t)$ depends only on the time difference $\tau=t_{2}-t_{1}$, i.e.,

$$
r_{\hat{\mu} \hat{\mu}}\left(t_{1}, t_{2}\right)=r_{\hat{\mu} \hat{\mu}}(\tau)
$$

where

$$
\begin{aligned}
r_{\hat{\mu} \hat{\mu}}\left(t_{1}, t_{2}\right) & =E\left\{\hat{\boldsymbol{\mu}}^{*}\left(t_{1}\right) \hat{\boldsymbol{\mu}}\left(t_{2}\right)\right\} \\
r_{\hat{\mu} \hat{\mu}}(\tau) & =E\left\{\hat{\boldsymbol{\mu}}^{*}(t) \hat{\boldsymbol{\mu}}(t+\tau)\right\} .
\end{aligned}
$$

Note that all SSS processes are WSS, but the converse need not be true. Following ([38], p. 422), we define a stochastic process $\hat{\mu}(t)$ to be asymptotically WSS if its mean is constant and its ACF $r_{\hat{\mu} \hat{\mu}}\left(t_{1}, t_{2}\right)$ depends only on the time difference $\tau=t_{2}-t_{1}$ as $t_{1} \rightarrow \pm \infty$ and/or $t_{2} \rightarrow \pm \infty$.

Furthermore, a stochastic process $\hat{\boldsymbol{\mu}}(t)$ is said to be mean ergodic if its ensemble average $m_{\hat{\mu}}$ equals the time average $m_{\tilde{\mu}}$ of $\tilde{\mu}(t)$, i.e.,

$$
m_{\hat{\mu}}=m_{\tilde{\mu}}:=\lim _{T \rightarrow \infty} \frac{1}{2 T} \int_{-T}^{T} \tilde{\mu}(t) d t .
$$

The stochastic process $\hat{\boldsymbol{\mu}}(t)$ is said to be autocorrelation ergodic if its ACF $r_{\hat{\mu} \hat{\mu}}(\tau)$ equals the time $\mathrm{ACF} r_{\tilde{\mu} \tilde{\mu}}(\tau)$ of $\tilde{\mu}(t)$, i.e.,

$$
r_{\hat{\mu} \hat{\mu}}(\tau)=r_{\tilde{\mu} \tilde{\mu}}(\tau):=\lim _{T \rightarrow \infty} \frac{1}{2 T} \int_{-T}^{T} \tilde{\mu}^{*}(t) \tilde{\mu}(t+\tau) d t .
$$

\section{Classification of channel simulators}

In this section, eight classes of SOC simulation models for frequency-nonselective Rayleigh fading channels will be introduced, seven of which are stochastic simulation models and one is completely deterministic. Before presenting a detailed analysis of the various classes of simulation models, some constraints are imposed on the three types of parameters. These constraints are as follows.

In cases, where the gains $\boldsymbol{c}_{n}$, frequencies $\boldsymbol{f}_{n}$, and phases $\boldsymbol{\theta}_{n}$ are random variables, it is reasonable to assume that their values are real and they are mutually independent. Also, it is assumed that the gains $\boldsymbol{c}_{1}, \boldsymbol{c}_{2}, \ldots, \boldsymbol{c}_{N}$ are i.i.d. random variables. The same is assumed for the sequences of random Doppler frequencies $\boldsymbol{f}_{1}, \boldsymbol{f}_{2}, \ldots, \boldsymbol{f}_{N}$ and phases $\boldsymbol{\theta}_{1}, \boldsymbol{\theta}_{2}, \ldots, \boldsymbol{\theta}_{N}$.

Whenever the gains $c_{n}$ and frequencies $f_{n}$ are constant quantities, it is assumed that they are different from 0 , such that $c_{n} \neq 0$ and $f_{n} \neq 0$ hold for all values of $n=1,2, \ldots, N$. Further constraints might also be imposed on the SOC model. For example, it is required that all frequencies $f_{n}$ are different. This latter condition is introduced to avoid correlations within the inphase (quadrature) component of $\tilde{\mu}(t)$.

\subsection{Class I channel simulators}

The channel simulators of Class I are defined by a set of deterministic processes $\tilde{\mu}(t)$ (see (5)) with constant gains $c_{n}$, constant frequencies $f_{n}$, and constant phases $\theta_{n}$. Since all model parameters are constants, there is no meaning to examine the stationary and ergodic properties of this class of channel simulators. However, in the following, the mean and ACF of $\tilde{\mu}(t)$ will be investigated. 
Since $\tilde{\mu}(t)$ is a deterministic process, its mean $m_{\tilde{\mu}}$ has to be determined using time averages instead of statistical averages. Using (5) and taking into account that $f_{n} \neq 0$, we obtain $m_{\tilde{\mu}}=0$. Hence, we realize that the deterministic process $\tilde{\mu}(t)$ of the simulation model has the same mean value as the stochastic process $\mu(t)$ of the reference model, i.e.,

$$
m_{\tilde{\mu}}=m_{\mu}=0 .
$$

Similarly, the ACF $r_{\tilde{\mu} \tilde{\mu}}(\tau)$ of $\tilde{\mu}(t)$ has to be determined using time averages. By taking into account that all frequencies $f_{n}$ are different, we can express the ACF $r_{\tilde{\mu} \tilde{\mu}}(\tau)$ of $\tilde{\mu}(t)$ in the following form

$$
r_{\tilde{\mu} \tilde{\mu}}(\tau)=\sum_{n=1}^{N} c_{n}^{2} e^{j 2 \pi f_{n} \tau}
$$

The mean $m_{\tilde{\mu}}$ and the ACF $r_{\tilde{\mu} \tilde{\mu}}(\tau)$ will be intensively used in the next subsections.

\subsection{Class II channel simulators}

The channel simulators of Class II are defined by a set of stochastic processes $\hat{\zeta}(t)$ with constant gains $c_{n}$, constant frequencies $f_{n}$, and random phases $\boldsymbol{\theta}_{n}$, which are uniformly distributed in the interval $(0,2 \pi]$. Using our notation, the complex process $\hat{\boldsymbol{\mu}}(t)=\hat{\boldsymbol{\mu}}_{1}(t)+j \hat{\boldsymbol{\mu}}_{2}(t)$ can be written as

$$
\hat{\boldsymbol{\mu}}(t)=\sum_{n=1}^{N} c_{n} e^{j\left(2 \pi f_{n} t+\boldsymbol{\theta}_{n}\right)} .
$$

In [22], it has been shown that the density $p_{\hat{\zeta}}(z)$ of the stochastic process $\hat{\zeta}(t)=|\hat{\boldsymbol{\mu}}(t)|$ can be represented as

$$
p_{\hat{\zeta}}(z)=(2 \pi)^{2} z \int_{0}^{\infty}\left[\prod_{n=1}^{N} J_{0}\left(2 \pi\left|c_{n}\right| y\right)\right] J_{0}(2 \pi z y) y d y .
$$

Also, in [22], it has been shown that the density $p_{\hat{\zeta}}(z)$ approaches the Rayleigh density as $N \rightarrow \infty$ and $c_{n}=$ $\sigma_{0} \sqrt{2 / N}$.

The mean $m_{\hat{\mu}}$ and the ACF $r_{\hat{\mu} \hat{\mu}}(\tau)$ of $\hat{\mu}(t)$ can be easily expressed as

$$
\begin{aligned}
m_{\hat{\mu}} & =0, \\
r_{\hat{\mu} \hat{\mu}}(\tau) & =\sum_{n=1}^{N} c_{n}^{2} e^{j 2 \pi f_{n} \tau} .
\end{aligned}
$$

With reference to (17), we notice that the density $p_{\hat{\zeta}}(z)$ is independent of time. Furthermore, from (18) and (19), we can realize that conditions 1 and 2 (see (8) and (9)) are fulfilled, respectively. Hence, the stochastic process $\hat{\zeta}(t)$ is FOS, and the stochastic process $\hat{\mu}(t)$ is WSS. A specific realization of the random phases $\boldsymbol{\theta}_{n}$ converts the stochastic process $\hat{\mu}(t)$ into a deterministic process (sample function) $\tilde{\mu}(t)$. This allows us to interpret Class I as a subset of Class II. The stochastic process $\hat{\mu}(t)$ is ME since the identity $m_{\hat{\mu}}=m_{\tilde{\mu}}$ holds. Also, the stochastic process $\hat{\boldsymbol{\mu}}(t)$ is AE. This statement follows from a comparison of (19) and (15), which reveals that the criterion $r_{\hat{\mu} \hat{\mu}}(\tau)=r_{\tilde{\mu} \tilde{\mu}}(\tau)$ is fulfilled.

\subsection{Class III channel simulators}

The channel simulators of Class III are defined by a set of stochastic processes $\hat{\zeta}(t)$ with constant gains $c_{n}$, random frequencies $\boldsymbol{f}_{n}$, and constant phases $\theta_{n}$. Hence, the complex process $\hat{\boldsymbol{\mu}}(t)=\hat{\boldsymbol{\mu}}_{1}(t)+j \hat{\boldsymbol{\mu}}_{2}(t)$ has the following form

$$
\hat{\boldsymbol{\mu}}(t)=\sum_{n=1}^{N} c_{n} e^{j\left(2 \pi \boldsymbol{f}_{n} t+\theta_{n}\right)}
$$

To obtain the density $p_{\hat{\zeta}}(z ; t)$ of the stochastic process $\hat{\zeta}(t)$, we continue as follows. In the first step, we consider a single complex cisoid at a fixed time instant $t=t_{0}$. Thus,

$$
\hat{\boldsymbol{\mu}}_{n}\left(t_{0}\right)=c_{n} e^{j\left(2 \pi \boldsymbol{f}_{n} t_{0}+\theta_{n}\right)}
$$

describes a complex random variable. From [29], in the limit $\left|t_{0}\right| \rightarrow \infty$, the density $p_{\hat{\mu}_{1, n}}\left(x_{1}\right)$ of $\hat{\boldsymbol{\mu}}_{1, n}\left(t_{0}\right)=$ $\operatorname{Re}\left\{\hat{\boldsymbol{\mu}}_{n}\left(t_{0}\right)\right\}=c_{n} \cos \left(2 \pi \boldsymbol{f}_{n} t_{0}+\theta_{n}\right)$ can be expressed as

$$
p_{\hat{\mu}_{1, n}}\left(x_{1}\right)= \begin{cases}\frac{1}{\pi\left|c_{n}\right| \sqrt{1-\left(x_{1} / c_{n}\right)^{2}}}, & \left|x_{1}\right|<c_{n} \\ 0, & \left|x_{1}\right| \geq c_{n} .\end{cases}
$$

Now, by following the same procedure as described in [22], we can conclude that the density $p_{\hat{\zeta}}(z ; \pm \infty)$ is given by (17). If $t$ is finite, we must replace the expression in (22) by ([29], Eq. (32)). In this case, it turns out that the density $p_{\hat{\zeta}}(z ; t)$ depends on time $t$ so that the stochastic process $\hat{\zeta}(t)$ is not FOS. Thus, we can conclude that a Class III channel simulator is an asymptotically FOS process.

In the following, we assume that the random frequencies $\boldsymbol{f}_{n}$ are given by

$$
\boldsymbol{f}_{n}=f_{\max } \cos \left(\boldsymbol{\alpha}_{n}\right)
$$

where the AOAs $\boldsymbol{\alpha}_{n}, n=1, \ldots, N$, are i.i.d. random variables, each having a density $p_{\alpha}(\alpha)$ identical to that characterizing the reference model's AOA statistics.

The mean $m_{\hat{\mu}}(t)$ of the stochastic process $\hat{\mu}(t)$ is obtained by computing the statistical average of (20) with respect to the random characteristics of the frequencies $\boldsymbol{f}_{n}$. Hence, we can write

$$
m_{\hat{\mu}}(t)=\frac{r_{\mu \mu}(t)}{2 \sigma_{0}^{2}} \sum_{n=1}^{N} c_{n} e^{j \theta_{n}},
$$

where $r_{\mu \mu}(t)$ is the ACF of the reference model described in (2). From (24), we see that the mean $m_{\hat{\mu}}(t)$ changes generally with time. To avoid this, we impose the following boundary condition on the phases 


$$
\sum_{n=1}^{N} c_{n} e^{j \theta_{n}}=0
$$

The condition above can easily be fulfilled if the number of cisoids $N$ is even; the gains $c_{n}$ are constants given by $c_{n}=\sigma_{0} \sqrt{2 / N}$, and $\theta_{n}=-\theta_{n+N / 2}=\pi / 2$ for $n=$ $1, \ldots, N / 2$. If the condition in (25) is fulfilled, then the mean $m_{\hat{\mu}}(t)$ is not only constant but also equal to zero, i.e., $m_{\hat{\mu}}=0$.

The ACF $r_{\hat{\mu} \hat{\mu}}\left(t_{1}, t_{2}\right)$ of $\hat{\boldsymbol{\mu}}(t)$ is obtained by substituting (20) in (10). In general, $r_{\hat{\mu} \hat{\mu}}\left(t_{1}, t_{2}\right)$ is time-shift sensitive since it is given as

$$
r_{\hat{\mu} \hat{\mu}}\left(t_{1}, t_{2}\right)=\sum_{n=1}^{N}\left[\frac{c_{n}^{2}}{2 \sigma_{0}^{2}} r_{\mu \mu}(\tau)+\frac{1}{4 \sigma_{0}^{4}} r_{\mu \mu}^{*}\left(t_{1}\right) r_{\mu \mu}\left(t_{2}\right) \sum_{\substack{m=1 \\ m \neq n}}^{N} c_{n} c_{m} e^{j\left(\theta_{n}-\theta_{m}\right)}\right] .
$$

However, $r_{\hat{\mu} \hat{\mu}}\left(t_{1}, t_{2}\right)$ becomes time-shift insensitive if we impose the following boundary condition on the gains and phases

$$
\sum_{n=1}^{N} \sum_{\substack{m=1 \\ m \neq n}}^{N} c_{n} c_{m} e^{j\left(\theta_{n}-\theta_{m}\right)}=0
$$

In case that the above boundary condition is fulfilled and the gains $c_{n}$ are equal to $c_{n}=\sigma_{0} \sqrt{2 / N}$, we easily see that the ACF $r_{\hat{\mu} \hat{\mu}}(\tau)$ of the stochastic simulation model is identical to the ACF $r_{\mu \mu}(\tau)$ of the reference model.

In Appendix 1, it is shown that conditions (25) and (27) cannot be simultaneously satisfied. However, if we let $t_{1} \rightarrow \pm \infty$ and/or $t_{2} \rightarrow \pm \infty$, then the ACFs $r_{\mu \mu}^{*}\left(t_{1}\right)$ and/or $r_{\mu \mu}\left(t_{2}\right)$ tend to zero, and hence the ACF $r_{\hat{\mu} \hat{\mu}}\left(t_{1}, t_{2}\right)$ depends only on the time difference $\tau=t_{2}-t_{1}$. In this case, together with the condition given in (25), we can conclude that the stochastic process $\hat{\mu}(t)$ is an asymptotically WSS process. Furthermore, if $t \rightarrow \pm \infty$, then $\hat{\boldsymbol{\mu}}(t)$ tends to an asymptotically FOS process. The stochastic process $\hat{\boldsymbol{\mu}}(t)$ is ME since the condition $m_{\hat{\mu}}=m_{\tilde{\mu}}$ is fulfilled. However, the stochastic processes $\hat{\mu}(t)$ of Class III channel simulators are always non-AE because the inequality $r_{\hat{\mu} \hat{\mu}}(\tau) \neq r_{\tilde{\mu} \tilde{\mu}}(\tau)$ holds.

\subsection{Class IV channel simulators}

The channel simulators of Class IV are defined by a set of stochastic processes $\hat{\zeta}(t)$ with constant gains $c_{n}$, random frequencies $\boldsymbol{f}_{n}$, and random phases $\boldsymbol{\theta}_{n}$, which are uniformly distributed in the interval $(0,2 \pi]$. In this case, the complex process $\hat{\boldsymbol{\mu}}(t)=\hat{\boldsymbol{\mu}}_{1}(t)+j \hat{\boldsymbol{\mu}}_{2}(t)$ is given by

$$
\hat{\boldsymbol{\mu}}(t)=\sum_{n=1}^{N} c_{n} e^{j\left(2 \pi \boldsymbol{f}_{n} t+\boldsymbol{\theta}_{n}\right)} .
$$

The density $p_{\hat{\zeta}}(z)$ of $\hat{\zeta}(t)=|\hat{\mu}(t)|$ is still given by (17), as the random frequencies $\boldsymbol{f}_{n}$ have no effect on the density $p_{\hat{\zeta}}(z)$ in (17). The mean $m_{\hat{\mu}}(t)$ is given by (18). If the gains $c_{n}$ are equal to $c_{n}=\sigma_{0} \sqrt{2 / N}$ and the frequencies $\boldsymbol{f}_{n}$ are given by (23), then it is straightforward to show that the ACF $r_{\hat{\mu} \hat{\mu}}(\tau)$ of the stochastic simulation model is identical to the ACF $r_{\mu \mu}(\tau)$ of the reference model, i.e., $r_{\hat{\mu} \hat{\mu}}(\tau)=$ $r_{\mu \mu}(\tau)$. The stochastic processes $\hat{\mu}(t)$ of Class IV channel simulators are non-AE since the inequality $r_{\hat{\mu} \hat{\mu}}(\tau) \neq$ $r_{\tilde{\mu} \tilde{\mu}}(\tau)$ holds.

\subsection{Class V channel simulators}

A Class V channel simulator is determined by a stochastic process $\hat{\zeta}(t)$ with random gains $\boldsymbol{c}_{n}$, constant frequencies $f_{n}$, and constant phases $\theta_{n}$. Thus, the complex process $\hat{\boldsymbol{\mu}}(t)=\hat{\boldsymbol{\mu}}_{1}(t)+j \hat{\boldsymbol{\mu}}_{2}(t)$ has the following form

$$
\hat{\boldsymbol{\mu}}(t)=\sum_{n=1}^{N} \boldsymbol{c}_{n} e^{j\left(2 \pi f_{n} t+\theta_{n}\right)} .
$$

In Appendix 2, it is shown that the density $p_{\hat{\zeta}}(z ; t)$ of the stochastic process $\hat{\zeta}(t)=|\hat{\boldsymbol{\mu}}(t)|$ is given by

$$
\begin{aligned}
p_{\hat{\zeta}}(z ; t)= & 2 \pi z \int_{0}^{2 \pi} \int_{0}^{\infty}\left[\prod_{n=1}^{N} \int_{-\infty}^{\infty}\right. \\
& \left.p_{c}(y) e^{j 2 \pi r y \cos \left(2 \pi f_{n} t+\theta_{n}-\theta\right)} d y\right] J_{0}(2 \pi z r) r d r d \theta
\end{aligned}
$$

where $p_{c}(\cdot)$ denotes the density of the gains $\boldsymbol{c}_{n}$. From the equation above, we realize that the density $p_{\hat{\zeta}}(z ; t)$ is a function of time. Hence, the stochastic process $\hat{\zeta}(t)$ is not an FOS process.

In the following, we will impose on the stochastic channel simulator that the i.i.d. random variables $\boldsymbol{c}_{n}$ have zero mean and variance $\sigma_{c}^{2}$, i.e., $m_{c}=E\left\{\boldsymbol{c}_{n}\right\}=0$ and $\sigma_{c}^{2}=$ $\operatorname{Var}\left\{\boldsymbol{c}_{n}\right\}=E\left\{\boldsymbol{c}_{n}^{2}\right\}$. Thus, for the mean $m_{\hat{\mu}}(t)$ of the stochastic process $\hat{\mu}(t)$, we obtain $m_{\hat{\mu}}(t)=0$. The ACF $r_{\hat{\mu} \hat{\mu}}\left(t_{1}, t_{2}\right)$ of $\hat{\boldsymbol{\mu}}(t)$ can be expressed as

$$
r_{\hat{\mu} \hat{\mu}}\left(t_{1}, t_{2}\right)=\sum_{n=1}^{N}\left[\left(\sigma_{c}^{2}+m_{c}^{2}\right) e^{j 2 \pi f_{n} \tau}+\sum_{\substack{m=1 \\ m \neq n}}^{N} m_{c}^{2} e^{j\left(2 \pi\left(f_{n} t_{2}-f_{m} t_{1}\right)+\theta_{n}-\theta_{m}\right)}\right] .
$$

By taking into account that the gains $c_{n}$ have zero mean, i.e., $m_{c=0}$, we obtain

$$
\begin{aligned}
r_{\hat{\mu} \hat{\mu}}\left(t_{1}, t_{2}\right) & =r_{\hat{\mu} \hat{\mu}}(\tau) \\
& =\sigma_{c}^{2} \sum_{n=1}^{N} e^{j 2 \pi f_{n} \tau} .
\end{aligned}
$$

Despite the fact that the ACF of $\hat{\mu}(t)$ depends only on the time difference $\tau=t_{2}-t_{1}$ if $m_{c}=0$, the stochastic process $\hat{\mu}(t)$ turns out to be non-AE as the inequality $r_{\hat{\mu} \hat{\mu}}(\tau) \neq r_{\tilde{\mu} \tilde{\mu}}(\tau)$ holds. 


\subsection{Class VI channel simulators}

The channel simulators of Class VI comprise a set of stochastic processes $\hat{\zeta}(t)$ with random gains $c_{n}$, constant frequencies $f_{n}$, and random phases $\boldsymbol{\theta}_{n}$. In this case, the complex process $\hat{\boldsymbol{\mu}}(t)=\hat{\boldsymbol{\mu}}_{1}(t)+j \hat{\boldsymbol{\mu}}_{2}(t)$ is of type

$$
\hat{\boldsymbol{\mu}}(t)=\sum_{n=1}^{N} \boldsymbol{c}_{n} e^{j\left(2 \pi f_{n} t+\boldsymbol{\theta}_{n}\right)} .
$$

In order to find the density $p_{\hat{\zeta}}(z)$ of $\hat{\zeta}(t)=|\hat{\boldsymbol{\mu}}(t)|$ of Class VI channel simulators, we make use of the conditional density $p_{\hat{\zeta}}\left(z \mid \boldsymbol{c}_{n}=c_{n}\right)$ of Class II channel simulators. The density $p_{\hat{\zeta}}(z)$ of Class VI channel simulators can then be obtained by averaging the conditional density $p_{\hat{\zeta}}\left(z \mid \boldsymbol{c}_{n}=c_{n}\right)$ over the distribution $p_{c}(y)$ of the $N$ i.i.d. random variables $\boldsymbol{c}_{1}, \boldsymbol{c}_{2}, \ldots, \boldsymbol{c}_{N}$, i.e.,

$$
\begin{aligned}
p_{\hat{\zeta}}(z) & =\int_{-\infty}^{\infty} \ldots \int_{-\infty}^{\infty} p_{\hat{\zeta}}\left(z \mid c_{1}=y_{1}, \ldots, c_{N}=y_{N}\right) \prod_{n=1}^{N} p_{c}\left(y_{n}\right) d y_{1} \cdots d y_{N} \\
& =(2 \pi)^{2} z \int_{0}^{\infty}\left[\int_{-\infty}^{\infty} p_{c}(y) J_{0}(2 \pi|y|) v d y\right]^{N} J_{0}(2 \pi z v) v d v .
\end{aligned}
$$

From (34), we can conclude that the density $p_{\hat{\zeta}}(z)$ is independent of time. Hence, the stochastic process $\hat{\zeta}(t)$ is FOS. The mean $m_{\hat{\mu}}(t)$ of the stochastic process $\hat{\mu}(t)$ is still given by (18) since the behavior of the random gains $c_{n}$ has no effect on the mean in (18).

The ACF $r_{\hat{\mu} \hat{\mu}}(\tau)$ of the stochastic process $\hat{\mu}(t)$ can be expressed as

$$
r_{\hat{\mu} \hat{\mu}}(\tau)=\left(\sigma_{c}^{2}+m_{c}^{2}\right) \sum_{n=1}^{N} e^{j 2 \pi f_{n} \tau} .
$$

Without imposing any specific distribution on the random gains $\boldsymbol{c}_{n}$, we can conclude that the stochastic process $\hat{\boldsymbol{\mu}}(t)$ is non-AE since the inequality $r_{\hat{\mu} \hat{\mu}}(\tau) \neq r_{\tilde{\mu} \tilde{\mu}}(\tau)$ holds.

\subsection{Class VII channel simulators}

This class of channel simulators involves all stochastic processes $\hat{\zeta}(t)=|\hat{\boldsymbol{\mu}}(t)|$ with random gains $\boldsymbol{c}_{n}$, random frequencies $\boldsymbol{f}_{n}$, and constant phases $\theta_{n}$, i.e.,

$$
\hat{\boldsymbol{\mu}}(t)=\sum_{n=1}^{N} \boldsymbol{c}_{n} e^{j\left(2 \pi \boldsymbol{f}_{n} t+\theta_{n}\right)}
$$

To obtain the density $p_{\hat{\zeta}}(z)$ of the stochastic process $\hat{\zeta}(t)$, we consider first the case $t \rightarrow \pm \infty$. The density $p_{\hat{\zeta}}(z)$ can be considered as the conditional density $p_{\hat{\zeta}}\left(z \mid \boldsymbol{c}_{n}=c_{n}\right)$ obtained for Class III channel simulators. By following the same procedure described in SubSection 6.6, we can conclude that the density $p_{\hat{\zeta}}(z)$ is given by (34). If $t$ is finite, we have to repeat the procedure described in SubSection 6.6 using ([29], Eq. (32)) instead of (22). In this case, it turns out that the density $p_{\hat{\zeta}}(z ; t)$ is a function of $t$. Hence, the stochastic processes $\hat{\zeta}(t)$ of Class VII channel simulators are asymptotically FOS processes.

The mean $m_{\hat{\mu}}(t)$ of this class of channel simulators is given by

$$
m_{\hat{\mu}}(t)=\frac{m_{c} r_{\mu \mu}(t)}{2 \sigma_{\mu}^{2}} \sum_{n=1}^{N} e^{j \theta_{n}} .
$$

It follows from (37) that the mean value is time independent if any of the boundary conditions $m_{c}=0, t \rightarrow \pm \infty$, or (25) are fulfilled.

Let us assume that the random frequencies $\boldsymbol{f}_{n}$ are given by (23). Then, the ACF $r_{\hat{\mu} \hat{\mu}}\left(t_{1}, t_{2}\right)$ of the stochastic process $\hat{\mu}(t)$ is obtained by computing the statistical average of (31) with respect to the random characteristics of the frequencies $\boldsymbol{f}_{n}$. Thus, we obtain

$$
r_{\hat{\mu} \hat{\mu}}\left(t_{1}, t_{2}\right)=\frac{N\left(\sigma_{c}^{2}+m_{c}^{2}\right)}{2 \sigma_{0}^{2}} r_{\mu \mu}(\tau)+\frac{m_{c}^{2}}{4 \sigma_{0}^{4}} r_{\mu \mu}^{*}\left(t_{1}\right) r_{\mu \mu}\left(t_{2}\right) \sum_{n=1}^{N} \sum_{\substack{m=1 \\ m \neq n}}^{N} e^{j\left(\theta_{n}-\theta_{m}\right)} .
$$

From the equation above, it follows that the ACF $r_{\hat{\mu} \hat{\mu}}\left(t_{1}, t_{2}\right)$ depends only on the time difference $\tau=t_{2}-t_{1}$ if we impose on this class of channel simulators any of the boundary conditions $m_{c}=0$ or (27). In addition, similar to the explanations given in SubSection 6.3, we can conclude that the ACF $r_{\hat{\mu} \hat{\mu}}\left(t_{1}, t_{2}\right)$ depends only on the time difference $\tau=t_{2}-t_{1}$ if $t_{1} \rightarrow \pm \infty$ and/or $t_{2} \rightarrow \pm \infty$. Furthermore, remember from SubSection 6.3 that the conditions (25) and (27) cannot be fulfilled simultaneously. If any of the boundary conditions $m_{c}=0$ or (27) is fulfilled and if $\sigma_{c}^{2}=2 \sigma_{0}^{2} / N-m_{c}^{2}$, then $r_{\hat{\mu} \hat{\mu}}\left(t_{1}, t_{2}\right)$ in (38) reduces to

$$
r_{\hat{\mu} \hat{\mu}}\left(t_{1}, t_{2}\right)=r_{\hat{\mu} \hat{\mu}}(\tau)=r_{\mu \mu}(\tau)
$$

which states that the channel simulators of Class VII and the reference model have identical correlation properties. However, the stochastic process $\hat{\boldsymbol{\mu}}(t)$ is non-AE since the inequality $r_{\hat{\mu}_{i} \hat{\mu}_{i}}(\tau) \neq r_{\tilde{\mu}_{i} \tilde{\mu}_{i}}(\tau)$ holds.

\subsection{Class VIII channel simulators}

The channel simulators of Class VIII are defined by a set of stochastic processes $\hat{\zeta}(t)=|\hat{\boldsymbol{\mu}}(t)|$ with random gains $\boldsymbol{c}_{n}$, random frequencies $\boldsymbol{f}_{n}$, and random phases $\boldsymbol{\theta}_{n}$, i.e.,

$$
\hat{\boldsymbol{\mu}}(t)=\sum_{n=1}^{N} \boldsymbol{c}_{n} e^{j\left(2 \pi \boldsymbol{f}_{n} t+\boldsymbol{\theta}_{n}\right)} .
$$

The density $p_{\hat{\zeta}}(z)$ of the stochastic process $\hat{\zeta}(t)$ is given by (34) because the random behavior of the frequencies $\boldsymbol{f}_{n}$ has no influence on $p_{\hat{\zeta}}(z)$. Hence, a Class VIII channel simulator is FOS.

For this class of channel simulators, it is straightforward to show that the mean $m_{\hat{\mu}}(t)$ of the stochastic process 
$\hat{\boldsymbol{\mu}}(t)$ is constant and equal to zero, i.e., $m_{\hat{\mu}}=0$. From (38), we can easily obtain the ACF $r_{\hat{\mu} \hat{\mu}}(\tau)$ for this class of channel simulators by taking into account the random characteristics of the phases $\boldsymbol{\theta}_{n}$, i.e.,

$$
r_{\hat{\mu} \hat{\mu}}(\tau)=\frac{N\left(\sigma_{c}^{2}+m_{c}^{2}\right)}{2 \sigma_{0}^{2}} r_{\mu \mu}(\tau) .
$$

Hence, $r_{\hat{\mu} \hat{\mu}}(\tau)=r_{\mu \mu}(\tau)$ holds if $\sigma_{c}^{2}=2 \sigma_{0}^{2} / N-m_{c}^{2}$. Note that in contrast to Class VII channel simulators, the ACF of Class VIII channel simulators only depends on the time difference $\tau=t_{2}-t_{1}$ even if $m_{c} \neq 0$. However, the channel simulators of Class VIII prove to be non-AE since the inequality $r_{\hat{\mu}_{i} \hat{\mu}_{i}}(\tau) \neq r_{\tilde{\mu}_{i} \tilde{\mu}_{i}}(\tau)$ holds.

\section{Extension with respect to frequency selectivity}

In this section, we extend our fundamental study on the stationarity and ergodicity of SOC simulation models for frequency-nonselective channels to SOC simulation models for frequency-selective channels. The time-variant impulse response, denoted by $\tilde{h}\left(\tau^{\prime}, t\right)$, of deterministic SOC simulation models for frequency-selective channels can be expressed in the equivalent complex baseband by the following ([8], Eq. (3.9))

$$
\tilde{h}\left(\tau^{\prime}, t\right)=\sum_{n=1}^{N} c_{n} e^{j\left(2 \pi f_{n} t+\theta_{n}\right)} \delta\left(\tau^{\prime}-\tau_{n}^{\prime}\right),
$$

where $\tau_{n}^{\prime}$ is the propagation delay of the $n$th path. For reasons of causality, we impose that $\tau_{n}^{\prime} \geq 0$ for $n=1, \ldots, N$. Furthermore, the time-variant channel transfer function, denoted by $\tilde{H}\left(f^{\prime}, t\right)$, is obtained by taking the Fourier transform of $\tilde{h}\left(\tau^{\prime}, t\right)$ with respect to $\tau^{\prime}$, i.e.,

$$
\begin{aligned}
\tilde{H}\left(f^{\prime}, t\right) & =\int_{-\infty}^{\infty} \tilde{h}\left(\tau^{\prime}, t\right) e^{-j 2 \pi f^{\prime} \tau^{\prime}} d \tau^{\prime} \\
& =\sum_{n=1}^{N} c_{n} e^{j\left(2 \pi f_{n} t+\theta_{n}-2 \pi f^{\prime} \tau_{n}^{\prime}\right)} .
\end{aligned}
$$

The time-variant channel transfer function $\tilde{H}\left(f^{\prime}, t\right)$ depends on four types of parameters, namely the gains $c_{n}$, the frequencies $f_{n}$, the phases $\theta_{n}$, and the propagation delays $\tau_{n}^{\prime}$. Each of these parameters can be defined either as a random variable or as a constant quantity. Hence, altogether, $2^{4}=16$ classes of SOC models for frequencyselective Rayleigh fading channels can now be defined. The ACF, denoted by $\tilde{r}_{H H}\left(f^{\prime}, f^{\prime}+v^{\prime} ; t, t+\tau\right)$, of the timevariant channel transfer function $\tilde{H}\left(f^{\prime}, t\right)$, defined by $([8]$, Eq. (7.144))

$$
\tilde{r}_{H H}\left(f^{\prime}, f^{\prime}+v^{\prime} ; t, t+\tau\right)=\lim _{T \rightarrow \infty} \frac{1}{2 T} \int_{-T}^{T} \tilde{H}^{*}\left(f^{\prime}, t\right) \tilde{H}\left(f^{\prime}+v^{\prime}, t+\tau\right) d t,
$$

is called the time-frequency correlation function. After substituting (43) in (44) and taking into account that all frequencies $f_{n}$ are supposed to be different, we obtain

$$
\tilde{r}_{H H}\left(\nu^{\prime}, \tau\right)=\sum_{n=1}^{N} c_{n}^{2} e^{j\left(2 \pi f_{n} \tau-2 \pi v^{\prime} \tau_{n}\right)} .
$$

From (45), we can easily express the frequency correlation function $\tilde{r}_{H H}\left(v^{\prime}\right)$ and the time correlation function $\tilde{r}_{H H}(\tau)$ as

$$
\begin{aligned}
\tilde{r}_{H H}\left(v^{\prime}\right) & =\tilde{r}_{H H}\left(v^{\prime}, 0\right) \\
& =\sum_{n=1}^{N} c_{n}^{2} e^{-j 2 \pi v \tau_{n}^{\prime}}
\end{aligned}
$$

and

$$
\begin{aligned}
\tilde{r}_{H H}(\tau) & =\tilde{r}_{H H}(0, \tau) \\
& =\sum_{n=1}^{N} c_{n}^{2} e^{j 2 \pi f_{n} \tau},
\end{aligned}
$$

respectively. We notice that the two expressions in (46) and (47) are dual to each other. The duality allows us to deduce the properties of the frequency correlation function $\tilde{r}_{H H}\left(v^{\prime}\right)$ directly from the properties of the time correlation function $\tilde{r}_{H H}(\tau)$ if we replace the deterministic (random) frequencies $f_{n}\left(\boldsymbol{f}_{n}\right)$ by deterministic (random) propagation delays $\tau_{n}^{\prime}\left(\boldsymbol{\tau}_{n}^{\prime}\right)$. After a straightforward investigation of the wide-sense stationarity, mean ergodicity, autocorrelation ergodicity with respect to both frequency and time, and the first-order stationarity of the envelope of the underlying random processes characterizing the 16 different classes of frequency-selective SOC simulators, we obtain the results presented in Table 1. For reasons of brevity, we have omitted the detailed calculations leading to the results in Table 1.

\section{Discussion and applications to the design of channel simulators}

Based on the results presented in the previous sections, we can analyze and compare the stationary and ergodic properties of the most relevant frequency-nonselective and frequency-selective SOC channel simulators proposed in the literature. In [39], Schulze proposed a stochastic SOC model with random delays, constant gains, random frequencies, and random phases to simulate wideband Rayleigh fading channels satisfying the wide-sense stationary uncorrelated scattering (WSSUS) condition ([40], Sec. 2.3). This model, which is inspired by the Monte Carlo approach for the simulation of physical and mathematical systems, was refined and further developed by Höher in [41]. The channel simulators by Schulze and Höher can be categorized into the 12th class of frequencyselective SOC simulation models. They are therefore characterized by a WSS and ME random process having an 
Table 1 Classes of frequency-selective SOC models and their stationary and ergodic properties

\begin{tabular}{|c|c|c|c|c|c|c|c|c|c|c|c|c|}
\hline \multirow[t]{2}{*}{ Class } & \multirow[t]{2}{*}{ Delays } & \multirow[t]{2}{*}{ Gains } & \multirow[t]{2}{*}{ Freq. } & \multirow[t]{2}{*}{ Phases } & \multicolumn{2}{|c|}{ WSS } & \multicolumn{2}{|c|}{ ME } & \multicolumn{3}{|c|}{$\mathrm{AE}$} & \multirow{2}{*}{$\frac{\text { FOS }}{\text { Time }}$} \\
\hline & & & & & Freq. & Time & Freq. & Time & Freq. & Time & Time-Freq. & \\
\hline । & Det. & Det. & Det. & Det. & - & - & - & - & - & - & - & - \\
\hline$\|$ & Det. & Det. & Det. & Rand. & Yes & Yes & Yes & Yes & Yes & Yes & Yes & Yes \\
\hline III & Det. & Det. & Rand. & Det. & $\mathrm{No} / \mathrm{Yes}^{\mathrm{c}}$ & $\mathrm{No} / \mathrm{Yes}^{\mathrm{c}}$ & $\mathrm{No} / \mathrm{Yes}^{\mathrm{c}}$ & $\mathrm{No} / \mathrm{Yes}^{\mathrm{c}}$ & $\mathrm{No} / \mathrm{Yes}^{c}$ & No & No & $\mathrm{No} / \mathrm{Yes}^{\mathrm{c}}$ \\
\hline IV & Det. & Det. & Rand. & Rand. & Yes & Yes & Yes & Yes & Yes & No & No & Yes \\
\hline V & Det. & Rand. & Det. & Det. & $\mathrm{No} / \mathrm{Yes}^{\mathrm{e}}$ & No/Yes ${ }^{e}$ & No/Yes ${ }^{e}$ & $\mathrm{No} / \mathrm{Yes}^{\mathrm{e}}$ & No & No & No & No \\
\hline VI & Det. & Rand. & Det. & Rand. & Yes & $\mathrm{No} / \mathrm{Yes}^{\mathrm{e}}$ & Yes & Yes & No & No & No & Yes \\
\hline VII & Det. & Rand. & Rand. & Det. & $\mathrm{No} / \mathrm{Yes}^{\mathrm{e}}$ & $\mathrm{No} / \mathrm{Yes}^{\mathrm{e}}$ & $\mathrm{No} / \mathrm{Yes}^{\mathrm{e}}$ & $\mathrm{No} / \mathrm{Yes}^{\mathrm{e}}$ & No & No & No & No/Yes ${ }^{c}$ \\
\hline VIII & Det. & Rand. & Rand. & Rand. & Yes & Yes & Yes & Yes & No & No & No & Yes \\
\hline IX & Rand. & Det. & Det. & Det. & $\mathrm{No} / \mathrm{Yes}^{\mathrm{a}}$ & $\mathrm{No} / \mathrm{Yes}^{\mathrm{a}}$ & $\mathrm{No} / \mathrm{Yes}^{\mathrm{a}}$ & $\mathrm{No} / \mathrm{Yes}^{\mathrm{a}}$ & No & $\mathrm{No} / \mathrm{Yes}^{\mathrm{a}}$ & No & $\mathrm{No} / \mathrm{Yes}^{\mathrm{a}}$ \\
\hline$x$ & Rand. & Det. & Det. & Rand. & Yes & Yes & Yes & Yes & No & Yes & No & $\mathrm{No} / \mathrm{Yes}^{\mathrm{b}}$ \\
\hline$X I$ & Rand. & Det. & Rand. & Det. & $\mathrm{No} / \mathrm{Yes}^{\mathrm{a}}$ & $\mathrm{No} / \mathrm{Yes}^{\mathrm{a}}$ & $\mathrm{No} / \mathrm{Yes}^{\mathrm{a} / \mathrm{c} / \mathrm{d}}$ & $\mathrm{No} / \mathrm{Yes}^{\mathrm{a} / \mathrm{c} / \mathrm{d}}$ & No & No & No & No/Yes ${ }^{c}$ \\
\hline XII & Rand. & Det. & Rand. & Rand. & Yes & Yes & Yes & Yes & No & No & No & Yes \\
\hline XIII & Rand. & Rand. & Det. & Det. & No/Yes ${ }^{a / e}$ & No/Yes ${ }^{a / e}$ & No/Yes ${ }^{\mathrm{a} / \mathrm{e}}$ & $\mathrm{No} / \mathrm{Yes}^{\mathrm{a} / \mathrm{e}}$ & No & No & No & No \\
\hline XIV & Rand. & Rand. & Det. & Rand. & Yes & Yes & Yes & Yes & No & No & No & Yes \\
\hline$x V$ & Rand. & Rand. & Rand. & Det. & $\mathrm{No} / \mathrm{Yes}^{\mathrm{a} / \mathrm{c} / \mathrm{e}}$ & $\mathrm{No} / \mathrm{Yes}^{\mathrm{a} / \mathrm{c} / \mathrm{e}}$ & $\mathrm{No} / \mathrm{Yes}^{\mathrm{a} / \mathrm{c} / \mathrm{d} / \mathrm{e}}$ & No/Yesed/c/d/e & No & No & No & No/Yes ${ }^{c}$ \\
\hline XVI & Rand. & Rand. & Rand. & Rand. & Yes & Yes & Yes & Yes & No & No & No & Yes \\
\hline
\end{tabular}

Freq., frequency; WSS, wide-sense stationary; ME, mean-ergodic; AE, autocorrelation ergodic; FOS, first-order stationary; Rand., random; Det., deterministic. ${ }^{a}$ Only in the limit $f^{\prime} \rightarrow \pm \infty{ }^{b}{ }^{b}$ Only in the limit $f^{\prime} \rightarrow 0 ;{ }^{\complement}$ Only in the limit $t \rightarrow \pm \infty ;{ }^{d}$ If the boundary condition $\sum_{n=1}^{N} C_{n} \exp \left\{\theta_{n}\right\}=0$ is satisfied; ${ }^{e}$ If the mean of the random gains is equal to zero. 
FOS envelope. However, from the results presented in Section 7, we can conclude that the channel simulators developed by Schulze and Höher are not AE. This is graphically demonstrated in Figure 1, where we present a comparison between the ensemble ACF and the time ACF of the channel simulator proposed in [41] with $N=20$ cisoids, $\sigma_{0}^{2}=1$, and $f_{\max }=100 \mathrm{~Hz}$. Here, we assume that all the delays are equal to zero so we only consider frequency-nonselective channels. The curves depicted in this figure were obtained by considering the simulation of a mobile fading channel satisfying the isotropic scattering condition. Unless stated otherwise, the same setup is considered for all simulation examples presented throughout this section. Figure 2 provides a comparison between the Rayleigh distribution and the envelope PDF of the SOC model in [41] evaluated at three randomly chosen observation time instants $t_{1}, t_{2}$, and $t_{3}$. The graphs shown in Figure 2 demonstrate that the envelope of the SOC simulation model is an FOS process.

A different type of frequency-nonselective SOC model was introduced in [42] by Crespo and Jimenez to simulate WSSUS channels by applying a harmonic decomposition technique to compute the model parameters. The SOC simulator considered in that paper is defined by cisoids with constant (null) phases, constant frequencies, and random gains, where it is assumed that the cisoids' gains have zero mean. The underlying simulation model is thus a Class V SOC process that satisfies the conditions for wide-sense stationarity and mean ergodicity. However, it does not fulfill the conditions for autocorrelation ergodicity, and its envelope is not an FOS process. This is numerically demonstrated in Figures 3 and 4, where we have again considered the simulation of a mobile fading

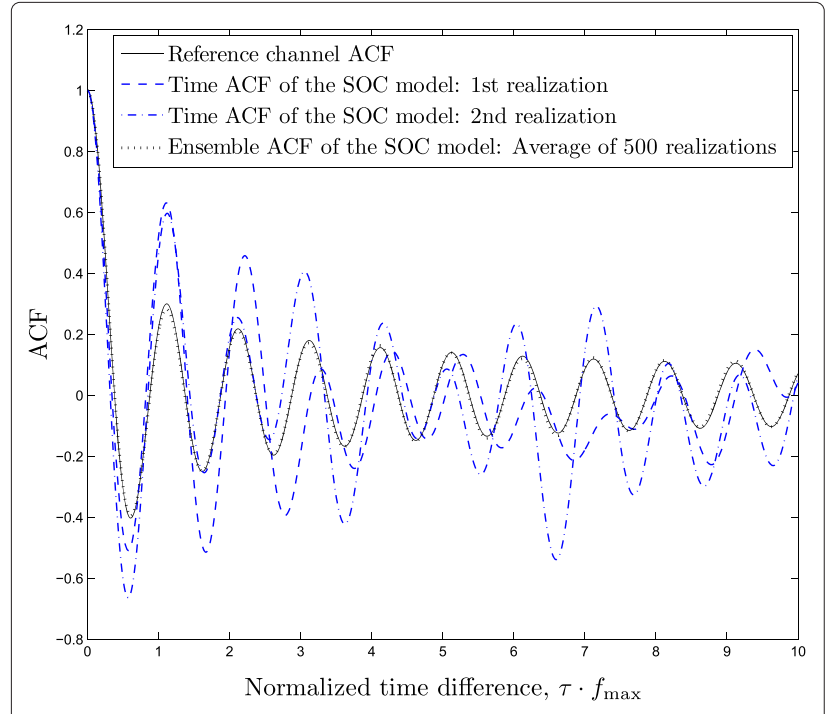

Figure 1 Ensemble and time ACFs of the Class III SOC channel simulator proposed in $[39,41]$.

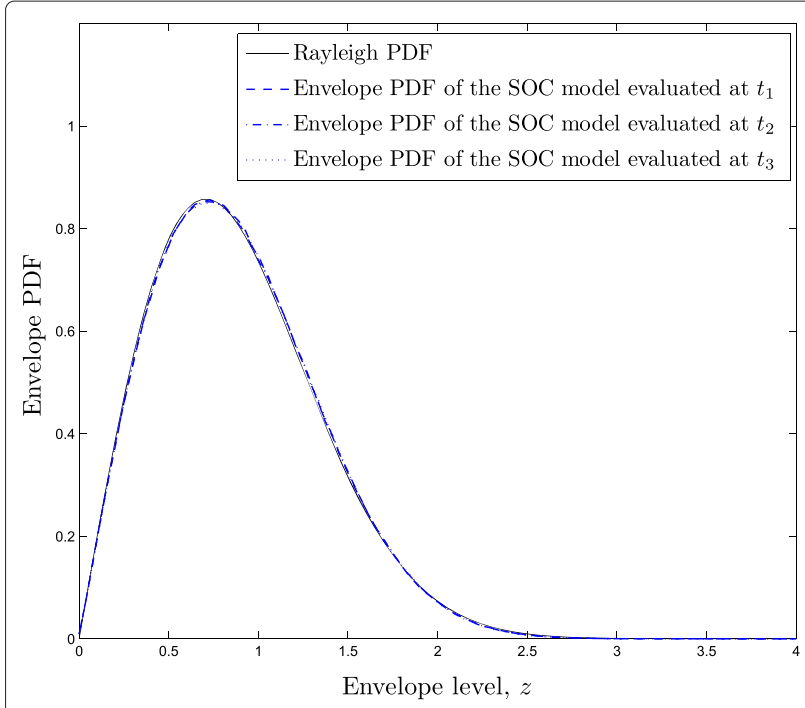

Figure 2 Comparison between Rayleigh distribution and envelope PDF of the Class III SOC channel simulator proposed in $[39,41]$.

channel under isotropic scattering conditions. The graphs presented in Figures 3 and 4 where obtained by setting the relevant parameters $p$ and $T$ of the method in [42] equal to $p=0.05$ and $T=20 / f_{\max }$. For this simulation setup, the resulting number of cisoids in SOC model equals $N=$ 281. It should be mentioned that the computational complexity by applying a harmonic decomposition technique has been addressed in [43].

The authors of [44] proposed a deterministic simulator for direction-selective and frequency-selective mobile radio channels. The generated waveforms of the simulator

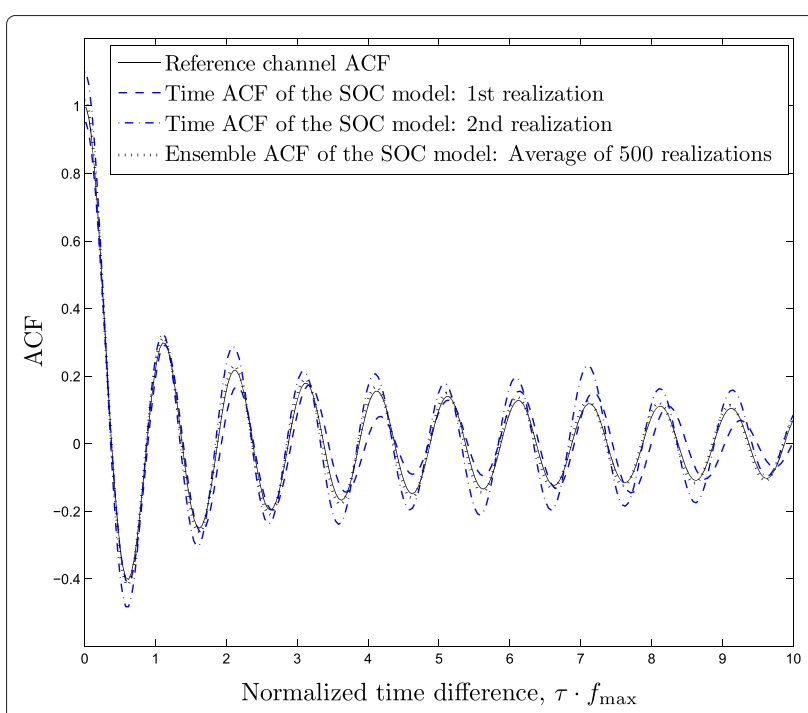

Figure 3 Ensemble and time ACFs of the Class V SOC channel simulator proposed in [42]. 


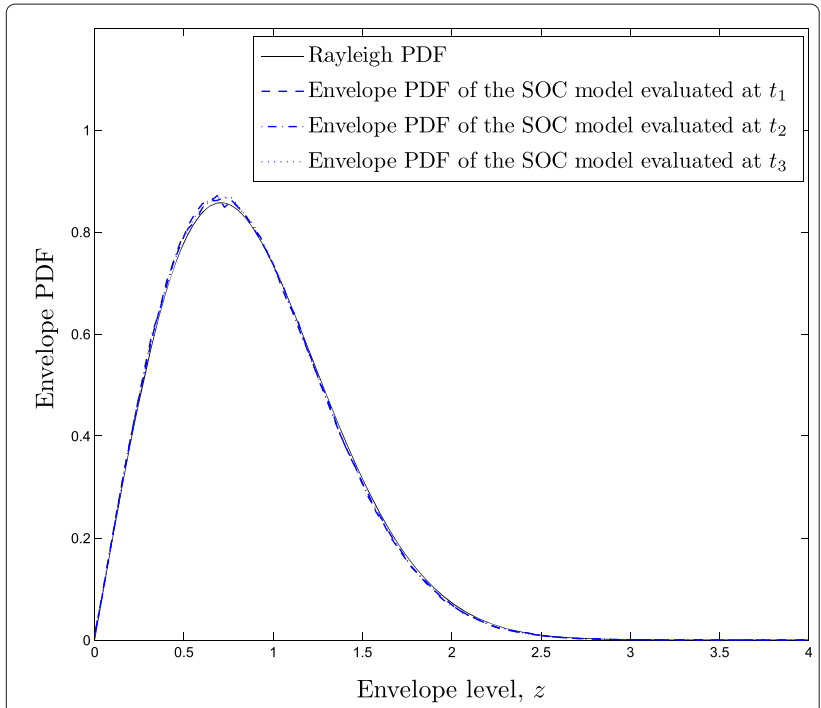

Figure 4 Comparison between Rayleigh distribution and envelope PDF of the Class V SOC channel simulator proposed in [42].

can be interpreted as sample functions of the frequencyselective Class II SOC model having cisoids with random phases uniformly distributed over the interval $(0,2 \pi]$. A channel simulator of frequency-nonselective or frequency-selective Class II SOC model corresponds in practice to scenarios where the scatterers around the receiver introduce constant gains and random phases. Furthermore, in these scenarios, the AOAs of the incoming waves are considered as constants due to the constant Doppler frequencies. Frequency-selective SOC channel models of Class II play an important role in the design of measurement-based channel simulators $[45,46]$. The correlation functions of such channel simulators match the correlation functions of measured channels accurately over a wide range determined by the length of the measured data. This can be achieved using the iterative nonlinear least square approximation method [47], which results in constant delays, constant gains, and constant Doppler frequencies, corresponding to constant AOAs. As the phases have no influence on the correlation functions, they are usually assumed to be i.i.d. uniform random variables. The assumption of constant AOAs has been applied in the well-known one-ring scattering model as demonstrated in [48,49]. Therein, the statistical properties of the presented channel simulator can be fitted nearly perfectly to the corresponding statistical properties of a reference model where the AOAs are continuous random variables with a given distribution. Hence, by considering constant AOAs in the channel simulator, we are able to simulate a large number of different propagation environments. From Sections 6 and 7, it follows that a random process characterizing a channel simulator as a frequency- nonselective or frequencyselective Class II SOC model is WSS, ME, and AE [49]. In addition, the envelope of the generated complex waveforms proves to be an FOS process. These observations are supported by the simulation results shown in Figures 5 and 6, where we present plots of the ensemble ACF, time ACF, and envelope PDF of the frequency-selective Class II SOC simulation model proposed in [44]. For the simulation results presented in Figures 5 and 6, we have again considered the case that all the delays are equal to zero.

Building upon the simulation models introduced in $[39,41,42,44]$, other researchers have developed new parameter computation methods to increase the simulator's accuracy and to reduce the simulation setup time. The characteristics of some noteworthy parameter computation methods are summarized in Table 2 . We refer the interested reader to $[8,26,36]$ for a detailed discussion on the performance of the parameter computation methods listed in Table 2.

Before closing this section, it is worth noticing that the stationary and ergodic properties of the eight classes of frequency-nonselective stochastic SOC and SOS models bear strong similarities as one may observe from the results presented in this paper and those obtained in [28-30]. A summary of the results obtained in this paper and those presented in [28-30] is given in Table 3.

This table shows that there exist some subtle but important differences between SOC and SOS models. For example, we have demonstrated that the SOC models of Class III are strictly non-WSS processes, whereas the SOS

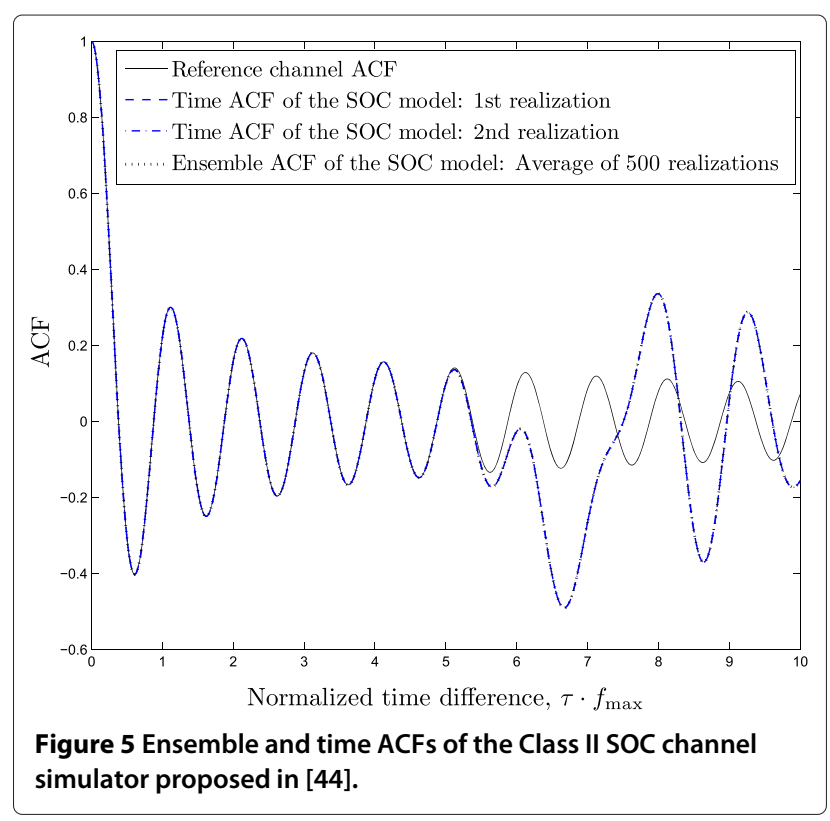




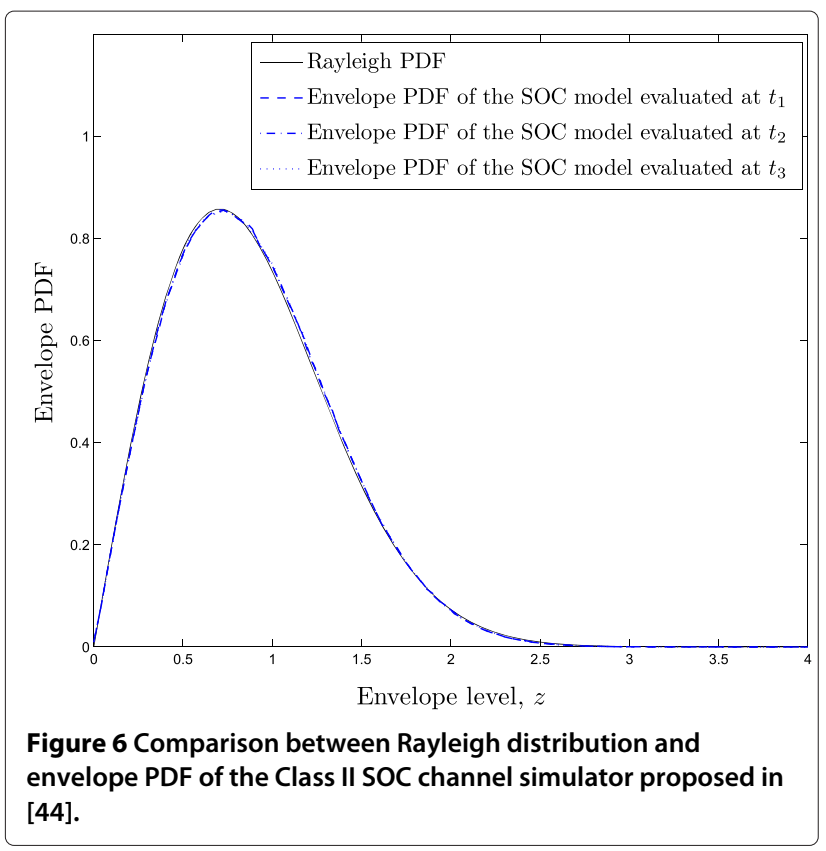

models of Class III are WSS if some boundary conditions are fulfilled [29]. On the other hand, we have shown that the SOC models of Class V are WSS if the random gains of the cisoids have zero mean. In contrast, it is demonstrated in [30] that the SOS models of Class V are strictly non-WSS.

\section{Conclusions}

In this paper, we provided a comprehensive analysis of the stationary and ergodic properties of eight classes of deterministic and stochastic SOC simulation models for frequency-nonselective mobile Rayleigh fading channels. We conclusively demonstrated that only the class defined by SOC models having cisoids with constant gains, constant Doppler frequencies, and random phases enables the design of channel simulators possessing the desired WSS, ME, AE, and FOS properties. In practice, this corresponds to scenarios where the scatterers around the receiver introduce constant gains and random phases. Furthermore, in these scenarios, the AOAs of the incoming waves are considered as constants due to the constant Doppler frequencies. Also, such a channel simulator can have nearly the same statistical properties as a given reference model where the AOAs are random variables with a given distribution. Furthermore, such a channel simulator that is extended to frequency-selective channels plays an important role in the design of measurementbased channel simulators. Hence, the channel simulator can be applied to a wide range of different propagation environments. The main focus of our work is on providing a solid and comprehensive framework that researchers can use to determine if a particular class of SOC models is well suited for the simulation of a given type of channel. For example, if one aims to simulate a fading channel having an FOS envelope, then it follows from the results presented in this paper that the SOC models of Class V are not a suitable option. A comparison with the results obtained in other papers for the stationary and ergodic properties of conventional SOS models shows that the stochastic SOC models and the SOS models have similar properties. However, there exist some important differences between the properties of both types of simulation models. Furthermore, we investigated the WSS, $\mathrm{ME}, \mathrm{AE}$, and FOS properties of SOC processes for the modeling and simulation of frequency-selective channels. Our investigations are of fundamental importance for the modeling and simulation of both SISO and MIMO channels.

The ergodic properties of Class II simulators for both frequency-nonselective and frequency-selective channels hold, provided that the lengths of the generated waveforms (sample functions) are infinite. Unfortunately, this condition cannot be fulfilled in practice. In order to determine whether the aforementioned properties hold under realistic simulation conditions, it is necessary to revisit the analysis presented in this paper by considering waveforms of finite length. We leave this important open problem for future research.

Table 2 Overview of parameter computation methods for SOC models and statistical properties of resulting channel simulators

\begin{tabular}{lcccc}
\hline Parameter computational method & Class & WSS & ME & AE \\
\hline Monte Carlo method [39,41] & IV & Yes & Yes & No \\
Harmonic decomposition technique [42] & V & Yes & Yes & No \\
Lp-norm method [44] & $\|$ & Yes & Yes & Yes \\
Method by Zheng [24] & IV & Yes & Yes & No \\
Extended method of exact Doppler spread [25] & $\|$ & Yes & Yes & Yes \\
Generalized method of equal areas [26] & $\|$ & Yes & Yes & Yes \\
Riemann sum method [36] & $\|$ & Yes & Yes & Yes \\
\hline
\end{tabular}

a FOS property refers to the envelope. 
Table 3 Classes of SOC and SOS models and their stationary and ergodic properties

\begin{tabular}{|c|c|c|c|c|c|c|c|}
\hline Class & Gains & Frequency & Phases & WSS & ME & $\mathrm{AE}$ & FOS \\
\hline I SOC & Det. & Det. & Det. & - & - & - & - \\
\hline I SOS & Det. & Det. & Det. & - & - & - & - \\
\hline II SOC & Det. & Det. & Rand. & Yes & Yes & Yes & Yes \\
\hline II SOS & Det. & Det. & Rand. & Yes & Yes & Yes & Yes \\
\hline III SOC & Det. & Rand. & Det. & $\mathrm{No} / \mathrm{Yes}^{\mathrm{a}, \mathrm{b}}$ & $\mathrm{No} / \mathrm{Yes}^{\mathrm{a}}$ & No & $\mathrm{No} / \mathrm{Yes}^{\mathrm{b}}$ \\
\hline III SOS & Det. & Rand. & Det. & $\mathrm{No} / \mathrm{Yes}^{\mathrm{c}, \mathrm{d}, \mathrm{e}}$ & $\mathrm{No} / \mathrm{Yes}^{\mathrm{c}, \mathrm{d}}$ & No & $\mathrm{No} / \mathrm{Yes}^{\mathrm{b}, \mathrm{c}, \mathrm{d}, \mathrm{e}}$ \\
\hline IV SOC & Det. & Rand. & Rand. & Yes & Yes & No & Yes \\
\hline IV SOS & Det. & Rand. & Rand. & Yes & Yes & No & Yes \\
\hline V SOC & Rand. & Det. & Det. & $\mathrm{No} / \mathrm{Yes}^{f}$ & $\mathrm{No} / \mathrm{Yes}^{\mathrm{f}}$ & No & No \\
\hline V SOS & Rand. & Det. & Det. & No & $\mathrm{No} / \mathrm{Yes}^{f}$ & No & No \\
\hline VISOC & Rand. & Det. & Rand. & Yes & Yes & No & Yes \\
\hline VISOS & Rand. & Det. & Rand. & Yes & Yes & No & Yes \\
\hline VII SOC & Rand. & Rand. & Det. & $\mathrm{No} / \mathrm{Yes}^{\mathrm{b} / \mathrm{f}}$ & $\mathrm{No} / \mathrm{Yes}^{\mathrm{a} / \mathrm{b} / \mathrm{f}}$ & No & No/Yes ${ }^{b}$ \\
\hline VII SOS & Rand. & Rand. & Det. & $\mathrm{No} / \mathrm{Yes}^{\mathrm{c}, \mathrm{d}, \mathrm{e}}$ & $\mathrm{No} / \mathrm{Yes}^{\mathrm{f} / c, \mathrm{~d}}$ & No & $\mathrm{No} / \mathrm{Yes}^{\mathrm{b}, c, \mathrm{~d}, \mathrm{e}}$ \\
\hline VIII SOC & Rand. & Rand. & Rand. & Yes & Yes & No & Yes \\
\hline VIII SOS & Rand. & Rand. & Rand. & Yes & Yes & No & Yes \\
\hline
\end{tabular}

WSS, wide-sense stationary; ME, mean-ergodic; $A E$, autocorrelation ergodic; FOS, first-order stationary; Rand., random; Det., deterministic. ${ }^{a}$ If the boundary condition $\sum_{n=1}^{N} c_{n} \exp \left\{j \theta_{n}\right\}=0$ is satisfied; ${ }^{\mathrm{b}}$ Only in the limit $t \rightarrow \pm \infty ;{ }^{\mathrm{c}}$ If the density of the random Doppler frequencies is an even function; ${ }^{\mathrm{d}}$ If the boundary condition $\sum_{n=1}^{N_{i}} \cos \left(\theta_{i, n}\right)=0$ is satisfied; ${ }^{\text {elf }}$ the boundary condition $\sum_{n=1}^{N_{i}} \cos \left(2 \theta_{i, n}\right)=0$ is satisfied; ${ }^{\mathrm{f}}$ If the mean value of the random gains is equal to zero.

\section{Appendix 1}

In this appendix, we will prove that the boundary conditions in (25) and (27) cannot be satisfied simultaneously. To that end, we observe that the equation in (27) is equivalent to

$$
\sum_{n=1}^{N} c_{n} e^{j \theta_{n}} \sum_{m=1}^{N} c_{m} e^{-j \theta_{m}}-\sum_{n=1}^{N} c_{n}^{2}=0 .
$$

Now, if (25) holds, then we have from (48) that

$$
\sum_{n=1}^{N} c_{n}^{2}=0 .
$$

The equation above poses a condition that cannot be satisfied because the cisoids' gains $c_{n}$ are different from zero, and therefore, $\sum_{n=1}^{N} c_{n}^{2}>0$. This concludes the proof.

\section{Appendix 2}

In the following, we derive the density function of a stochastic process $\hat{\zeta}(t)$ with random gains $c_{n}$, constant frequencies $f_{n}$, and constant phases $\theta_{n}$. The starting point for the derivation of the density of the stochastic process $\hat{\zeta}(t)=|\hat{\mu}(t)|$ is a single complex cisoid of the form

$$
\hat{\boldsymbol{\mu}}_{n}(t)=\boldsymbol{c}_{n} e^{j\left(2 \pi f_{n} t+\theta_{n}\right)} .
$$

For fixed values of $t=t_{0}, \hat{\mu}_{1, n}\left(t_{0}\right)=\operatorname{Re}\left\{\hat{\boldsymbol{\mu}}_{n}(t)\right\}=$ $\boldsymbol{c}_{n} \cos \left(2 \pi f_{n} t_{0}+\theta_{n}\right)$ and $\hat{\boldsymbol{\mu}}_{2, n}\left(t_{0}\right)=\operatorname{Im}\left\{\hat{\boldsymbol{\mu}}_{n}(t)\right\}=$ $\boldsymbol{c}_{n} \sin \left(2 \pi f_{n} t_{0}+\theta_{n}\right)$ represent two dependent random variables. From ([9], Eq. (5-18)), the density $p_{\hat{\mu}_{1, n}}\left(x_{1} ; t_{0}\right)$ of $\hat{\boldsymbol{\mu}}_{1, n}\left(t_{0}\right)$ can be expressed as

$$
p_{\hat{\mu}_{1, n}}\left(x_{1} ; t_{0}\right)=\frac{p_{c}\left(x_{1} / \cos \left(2 \pi f_{n} t_{0}+\theta_{n}\right)\right)}{\left|\cos \left(2 \pi f_{n} t_{0}+\theta_{n}\right)\right|},
$$

where $p_{c}(\cdot)$ denotes the common density of the gains $\boldsymbol{c}_{n}$. Using ([50], Eq. (3.15)), the joint density $p_{\hat{\mu}_{1, n} \hat{\mu}_{2, n}}\left(x_{1}, x_{2} ; t_{0}\right)$ of the dependent random variables $\hat{\boldsymbol{\mu}}_{1, n}\left(t_{0}\right)$ and $\hat{\boldsymbol{\mu}}_{2, n}\left(t_{0}\right)$ can be obtained as

$$
p_{\hat{\mu}_{1, n} \hat{\mu}_{2, n}}\left(x_{1}, x_{2} ; t_{0}\right)=p_{\hat{\mu}_{1, n}}\left(x_{1} ; t_{0}\right) \delta\left(x_{2}-g\left(x_{1} ; t_{0}\right)\right)
$$

where $g\left(x_{1} ; t_{0}\right)=x_{1} \tan \left(2 \pi f_{n} t_{0}+\theta_{n}\right)$. The joint characteristic function $\Psi_{\hat{\mu}_{1, n} \hat{\mu}_{2, n}}\left(\nu_{1}, \nu_{2} ; t_{0}\right)$ of the random variables $\hat{\boldsymbol{\mu}}_{1, n}\left(t_{0}\right)$ and $\hat{\boldsymbol{\mu}}_{2, n}\left(t_{0}\right)$ is defined by the Fourier transformation

$$
\begin{gathered}
\Psi_{\hat{\mu}_{1, n} \hat{\mu}_{2, n}}\left(v_{1}, v_{2} ; t_{0}\right)=\int_{-\infty}^{\infty} \int_{-\infty}^{\infty} p_{\hat{\mu}_{1, n} \hat{\mu}_{2, n}} \\
\left(x_{1}, x_{2} ; t_{0}\right) e^{j 2 \pi\left(\nu_{1} x_{1}+\nu_{2} x_{2}\right)} d x_{1} d x_{2} .
\end{gathered}
$$

After substituting (52) in (53) and carrying out some algebraic computations, we find

$$
\begin{gathered}
\Psi_{\hat{\mu}_{1, n} \hat{\mu}_{2, n}}\left(v_{1}, v_{2} ; t_{0}\right)=\int_{-\infty}^{\infty} p_{c}\left(x_{1}\right) e^{j 2 \pi v_{1} x_{1} \cos \left(2 \pi f_{n} t_{0}+\theta_{n}\right)} \\
e^{j 2 \pi v_{2} x_{1} \sin \left(2 \pi f_{n} t_{0}+\theta_{n}\right)} d x_{1} .
\end{gathered}
$$


Since we have assumed that the gains $c_{n}$ are i.i.d. random variables, it follows that the quantities $\boldsymbol{\mu}_{n}\left(t_{0}\right)$ are also i.i.d. random variables. Hence, the joint characteristic function $\Psi_{\hat{\mu}_{1} \hat{\mu}_{2}}\left(v_{1}, v_{2} ; t_{0}\right)$ of $\hat{\mu}_{1}\left(t_{0}\right)$ and $\hat{\mu}_{2}\left(t_{0}\right)$ can be expressed as the product of the joint characteristic functions $\Psi_{\hat{\mu}_{1, n} \hat{\mu}_{2, n}}\left(v_{1}, v_{2} ; t_{0}\right)$ of $\hat{\boldsymbol{\mu}}_{1, n}\left(t_{0}\right)$ and $\hat{\boldsymbol{\mu}}_{2, n}\left(t_{0}\right)$, i.e.,

$$
\Psi_{\hat{\mu}_{1} \hat{\mu}_{2}}\left(v_{1}, v_{2} ; t_{0}\right)=\prod_{n=1}^{N} \Psi_{\hat{\mu}_{1, n} \hat{\mu}_{2, n}}\left(v_{1}, v_{2} ; t_{0}\right) .
$$

From this and the two-dimensional inversion formula for Fourier transforms, it follows that the joint density $p_{\hat{\mu}_{1} \hat{\mu}_{2}}\left(x_{1}, x_{2} ; t_{0}\right)$ of the statistically dependent random variables $\hat{\mu}_{1}\left(t_{0}\right)$ and $\hat{\mu}_{2}\left(t_{0}\right)$ can be expressed as

$$
\begin{aligned}
p_{\hat{\mu}_{1} \hat{\mu}_{2}}\left(x_{1}, x_{2} ; t_{0}\right)= & \int_{-\infty}^{\infty} \int_{-\infty}^{\infty}\left[\prod_{n=1}^{N} \int_{-\infty}^{\infty} p_{c}(y) e^{j 2 \pi v_{1} y \cos \left(2 \pi f_{n} t_{0}+\theta_{n}\right)}\right. \\
\times & \left.e^{j 2 \pi v_{2} y \sin \left(2 \pi f_{n} t_{0}+\theta_{n}\right)} d y\right] \\
& e^{-j 2 \pi\left(\nu_{1} x_{1}+v_{2} x_{2}\right)} d \nu_{1} d v_{2}
\end{aligned}
$$

If we now transform the Cartesian coordinates $\left(x_{1}, x_{2}\right)$ into polar coordinates $(z, \varphi)$ by means of $x_{1}=z \cos \varphi$ and $x_{2}=z \sin \varphi$, then we obtain the joint density $p_{\hat{\zeta} \hat{\vartheta}}\left(z, \varphi ; t_{0}\right)$ of the envelope $\hat{\boldsymbol{\zeta}}\left(t_{0}\right)=\left|\hat{\boldsymbol{\mu}}\left(t_{0}\right)\right|$ and the phase $\hat{\boldsymbol{\vartheta}}\left(t_{0}\right)=$ $\arg \left\{\hat{\boldsymbol{\mu}}\left(t_{0}\right)\right\}$ as

$$
\begin{aligned}
p_{\hat{\zeta} \hat{\vartheta}}\left(z, \varphi ; t_{0}\right)= & z p_{\hat{\mu}_{1} \hat{\mu}_{2}}\left(z \cos \varphi, z \sin \varphi ; t_{0}\right) \\
= & z \int_{-\infty}^{\infty} \int_{-\infty}^{\infty}\left[\prod_{n=1}^{N} \int_{-\infty}^{\infty} p_{c}(y) e^{j 2 \pi v_{1} y \cos \left(2 \pi f_{n} t_{0}+\theta_{n}\right)}\right. \\
& \left.\quad \times e^{j 2 \pi \nu_{2} y \sin \left(2 \pi f_{n} t_{0}+\theta_{n}\right)} d y\right] \\
& \times e^{-j 2 \pi z\left(\nu_{1} \cos \varphi+\nu_{2} \sin \varphi\right)} d \nu_{1} d \nu_{2},
\end{aligned}
$$

for $z \geq 0$ and $\varphi \in(-\pi, \pi)$. Finally, the density $p_{\hat{\zeta}}\left(z ; t_{0}\right)$ of the envelope $\hat{\zeta}\left(t_{0}\right)$ can be obtained from the joint density $p_{\hat{\zeta} \hat{\vartheta}}\left(z, \theta ; t_{0}\right)$ by integrating over $\varphi$. By following this procedure, using ([51], Eq. (3.937.2)) and transforming the Cartesian coordinates $\left(v_{1}, v_{2}\right)$ into polar coordinates $(r, \theta)$, we finally obtain

$$
\begin{array}{r}
p_{\hat{\zeta}}\left(z ; t_{0}\right)=2 \pi z \int_{0}^{2 \pi} \int_{0}^{\infty}\left[\prod_{n=1}^{N} \int_{-\infty}^{\infty} p_{c}(y) e^{j 2 \pi r y \cos \left(2 \pi f_{n} t_{0}+\theta_{n}-\theta\right)}\right. \\
d y] J_{0}(2 \pi z r) r d r d \theta .
\end{array}
$$

\section{Endnotes}

${ }^{a}$ We notice, nonetheless, that the conventional SOS simulation approach can be applied to the simulation of fading channels with asymmetrical DPSDs if the conventional approach is used in connection with the Hilbert transform ([8], Sec. 6.1.4).

${ }^{\mathrm{b}}$ For clarity, we will use bold letters to indicate stochastic processes as well as random variables, and normal letters are used for deterministic processes (sample functions) and realizations (outcomes) of random variables.

\section{Competing interests}

The authors declare that they have no competing interests.

\section{Acknowledgements}

This paper was presented in parts at IEEE 67th Vehicular Technology Conference, VTC2008-Spring, Singapore, May 2008, and European Wireless Conference, Vienna, Austria, April 2011. This work was supported in part by the Basque Government through the INFOLIMITS project (PI2012-10), and by the Spanish Ministry of Science and Innovation through the projects COSIMA(TEC2010-19545-C04-02) and COMONSENS (CSD2008-00010).

\section{Author details}

${ }^{1}$ CEIT and Tecnun, University of Navarra, Manuel de Lardizábal 15, San Sebastián 20018, Spain. ² Faculty of Science, Universidad Autonoma de San Luis Potosi, San Luis Potosi 78290, Mexico. ${ }^{3}$ Faculty of Engineering and Science, University of Agder, Servicebox 509, Grimstad NO-4898, Norway.

Received: 6 September 2012 Accepted: 19 April 2013

Published: 10 May 2013

\section{References}

1. BH Fleury, PE Leuthold, Radiowave propagation in mobile communications: an overview of European research. IEEE Commun. Mag. 34, 70-81 (1996)

2. B Sklar, Digital Communications: Fundamentals and Applications, 2nd edn, chap. 15. (Prentice Hall, New Jersey, 2001), pp. 944-1011

3. P Sharma, Time-series model for wireless fading channels in isotropic and non-isotropic scattering environments. IEEE Commun. Lett. 9, 46-48 (2005)

4. II Smith, A computer generated multipath fading simulation for mobile radio. IEEE Trans. Veh. Technol. 24(3), 39-40 (1975)

5. RB Ertel, JH Reed, Generation of two equal power correlated Rayleigh fading envelopes. IEEE Commun. Lett. 2(10), 276-278 (1998)

6. KW Yip, TS Ng, Karhunen-Loève expansion of the WSSUS channel output and its application to efficient simulation. IEEE J. Select. Areas Commun. 15(4), 640-646 (1997)

7. GL Stüber, Principles of Mobile Communication, 3rd edn. (Kluwer Academic Publishers, Boston, 2011)

8. M Pätzold, Mobile Radio Channels, 2nd edn. (John Wiley and Sons, Chichester, 2011)

9. A Papoulis, SU Pillai, Probability, Random Variables and Stochastic Processes, 4th edn. (McGraw-Hill, New York, 2002)

10. M Pätzold, F Laue, The performance of deterministic Rayleigh fading channel simulators with respect to the bit error probability. Vehicular Technology Conference Proceedings, VTC 2000-Spring, Tokyo, IEEE. Piscataway, 1998-2003 (2000)

11. Y Ma, M Pätzold, Performance analysis of wideband sum-of-cisoids-based channel simulators with respect to the bit error probability of DPSK OFDM systems. Vehicular Technology Conference Proceedings, VTC 2009-Spring, Barcelona IEEE. Piscataway, 1-6 (2009)

12. BH Fleury, An uncertainty relation for WSS processes and its application to WSSUS systems. IEEE Trans. Commun. 44, 1632-1634 (1996)

13. A Paier, T Zemen, L Bernado, G Matz, J Karedal, N Czink, C Dumard, F Tufvesson, AF Molisch, CF Mecklenbräuker, Non-WSSUS vehicular channel characterization in highway and urban scenarios at $5.2 \mathrm{GHz}$ using 
the local scattering function. International Workshop on Smart Antennas, (WSA 2008), 9-15. Darmstadt (IEEE, Piscataway, 2008)

14. D Umansky, M Pätzold, in IEEE Global Communications Conference Proceedings GLOBECOM. Stationarity test for wireless communication channels (IEEE, Piscataway, Honolulu, 2009), pp. 1-6

15. SO Rice, Mathematical analysis of random noise. Bell Syst. Tech. J. 23, 282-332 (1944)

16. SO Rice, Mathematical analysis of random noise. Bell Syst. Tech. J. 24, 46-156 (1945)

17. WC Jakes, Microwave Mobile Communications. (IEEE Press, Piscataway, 1994)

18. M Pätzold, U Killat, F Laue, Deterministic digital simulation model for Suzuki processes with application to a shadowed Rayleigh land mobile radio channel. IEEE Trans. Veh. Technol. 45(2), 318-331 (1996)

19. CXiao, J Wu, SY Leong, YR Zheng, KB Letaief, A discrete-time model for triply selective MIMO Rayleigh fading channels. IEEE Trans. Wireless Commun. 3(5), 1678-1688 (2004)

20. CS Patel, GL Stüber, TG Pratt, Comparative analysis of statistical models for the simulation of Rayleigh faded cellular channels. IEEE Trans. Commun. 53(6), 1017-1026 (2005)

21. A Alimohammad, BF Cockburn, Modeling and hardware implementation aspects of fading channel simulators. IEEE Trans. Veh. Technol. 57(4), 2055-2069 (2008)

22. M Pätzold, B Talha, On the statistical properties of sum-of-cisoids-based mobile radio channel models, in Proceeding of the 10th International Symposium on Wireless Personal Multimedia Communications, WPMC. Jaipur, 394-400 (2007)

23. CA Gutiérrez, Channel Simulation Models for Mobile Broadband Communication Systems. (University of Agder, Doctoral Dissertation, 2009)

24. YR Zheng, in Proceedings of the 2006 IEEE Conference on Military Communications (MILCOM'06), Washington, DC. A non-isotropic model for mobile-to-mobile fading channel simulations (IEEE, Piscataway, 2006), pp. 1-7

25. M Pätzold, BO Hogstad, N Youssef, Modeling, analysis, and simulation of MIMO mobile-to-mobile fading channels. IEEE Trans. Wireless Commun. 7(2), 510-520 (2008)

26. CA Gutiérrez, M Pätzold, The generalized method of equal areas for the design of sum-of-cisoids simulators for mobile Rayleigh fading channels with arbitrary Doppler spectra. Wirel. Commun. Mob. Comput. (2011). 10.1002/wcm. 1154

27. GD Durgin, Space-Time Wireless Channels. (Prentice Hall, New Jersey, 2003)

28. M Pätzold, in Proceeding of the 14th IEEE International Symposium on Personal, Indoor and Mobile Radio Communications, PIMRC 2003. On the stationarity and ergodicity of fading channel simulators basing on Rice's sum-of-sinusoids (IEEE, Piscataway Beijing, 2003), pp. 1521-1525

29. M Pätzold, BO Hogstad, Classes of sum-of-sinusoids Rayleigh fading channel simulators and their stationary and ergodic properties-part I. WSEAS Trans. on Math. 5, 222-230 (2006)

30. M Pätzold, BO Hogstad, Classes of sum-of-sinusoids Rayleigh fading channel simulators and their stationary and ergodic properties-part II. WSEAS Trans. Math. 4(4), 441-449 (2005)

31. CA Gutiérrez, M Pätzold, On the correlation and ergodic properties of the squared envelope of SOC Rayleigh fading channel simulators. Wireless Personal Commun. 68(3), 963-979 (2013)

32. M Pätzold, U Killat, F Laue, An extended Suzuki model for land mobile satellite channels and its statistical properties. IEEE Trans. Veh. Technol. 47(2), 617-630 (1998)

33. FD Neeser, JL Massey, Proper complex random processes with applications to information theory. IEEE Trans. Inform. Theory. 39(4), 1293-1302 (1993)

34. RH Clarke, A statistical theory of mobile-radio reception. Bell Syst. Tech. J. 47, 957-1000 (1968)

35. CA Gutiérrez, M Pätzold, in Proceedings of the 50th IEEE Global Telecommunications Conference, GLOBECOM 2007.

Sum-of-sinusoids-based simulation of flat fading wireless propagation channels under non-isotropic scattering conditions (IEEE, Piscataway Washington DC, 2007), pp. 3842-3846

36. CA Gutiérrez, M Pätzold, The design of sum-of-cisoids Rayleigh fading channel simulators assuming non-isotropic scattering conditions. IEEE Trans. Wireless Commun. 9(4), 1308-1314 (2010)
37. X Cheng, CX Wang, DI Laurenson, S Salous, AV Vasilakos, New deterministic and stochastic simulation models for non-isotropic scattering mobile-to-mobile Rayleigh fading channels. Wireless Commun. and Mobile Computing 11(7), 829-842 (July 2011)

38. JJ Shynk, Probability, Random Variables, and Random Processes: Theory and Signal Processing Applications, 1st edn. (Wiley-Interscience, Hoboken, 2012)

39. H Schulze, in Proceedings of, Kleinheubacher Reports of the German PTT, U.R.S.I/ITG Conference in Kleinheubach. Stochastische Modelle und digitale Simulation von Mobilfunkkanälen, vol.32 (Darmstadt, 1988), pp. 473-483

40. H Schulze, L Lüders, Theory and Applications of OFDM and CDMA. (John Wiley and Sons, Chichester, 2005)

41. P Höher, A statistical discrete-time model for the WSSUS multipath channel. IEEE Trans. Veh. Technol. 41(4), 461-468 (1992)

42. P Crespo, J Jiménez, Computer simulation of radio channels using a harmonic decomposition technique. IEEE Trans. Veh. Technol. 44(3), 414-419 (1995)

43. R Parra-Michel, VY Kontorovich, AG Orozco-Lugo, M Lara, in Proceedings of IEEE 58th Vehicular Technology Conference, VTC 2003-Fall, Orlando, Computational complexity of narrow band and wide band channel simulators (IEEE, Piscataway, 2003), pp. 143-148

44. M Pätzold, N Youssef, Modelling and simulation of direction-selective and frequency-selective mobile radio channels. Int. J. Electron. Commun. AEÜ-55(6), 433-442 (2001)

45. D Umansky, M Pätzold, in Proceedings of the 4th IEEE International Symposium on Wireless Communication Systems, ISWCS 2007, Trondheim. Design of measurement-based wideband mobile radio channel simulators (IEEE, Piscataway, 2007), pp. 229-235

46. D Umansky, M Pätzold, in Proceedings of the 67th IEEE Vehicular Technology Conference, VTC 2008-Spring, Singapore. Design of wideband mobile radio channel simulators based on real-world measurement data (IEEE, Piscataway, 2008), pp. 319-324

47. A Fayziyev, M Pätzold, An improved iterative nonlinear least square approximation method for the design of SISO wideband mobile radio channel simulators. REV J. Electron. Commun. 2(1-2), 19-25 (2012)

48. TA Chen, MP Fitz, WY Kuo, MD Zoltowski, JH Grimm, A space-time model for frequency nonselective Rayleigh fading channels with applications to space-time modems. IEEE J. Select. Areas Commun. 18(7), 1175-1190 (2000)

49. M Pätzold, BO Hogstad, A space-time simulator for MIMO channels based on the geometrical one-ring scattering model. Wirel. Commun. Mob. Comput. 4, 727-737 (2004)

50. S Primak, VY Kontorovich, V Lyandres, Stochastic Methods and their Applications to Communications - Stochastic Differential Equations Approach. (John Wiley \& Sons, Chichester, 2004)

51. IS Gradshteyn, IM Ryzhik, Tables of Series, Products, and Integrals, Volume I and II, 5th edn. (Harri Deutsch, Frankfurt, 1981)

\section{doi:10.1186/1687-1499-2013-125}

Cite this article as: Hogstad et al:: Classes of sum-of-cisoids processes and their statistics for the modeling and simulation of mobile fading channels. EURASIP Journal on Wireless Communications and Networking 2013 2013:125. 